\title{
TECHNICAL SOLUTIONS TO MITIGATE SHIFTING FISH FAUNA ZONES IMPACTED BY LONG TERM HABITAT DEGRADATION IN THE BISTRA MĂRUI RIVER - STUDY CASE
}

\author{
Doru BĂNĂDUC *, Răzvan VOICU **, Lee Jason BAUMGARTNER ***,

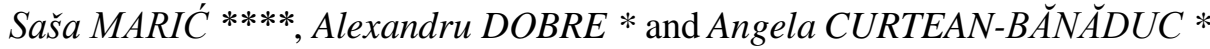 \\ * "Lucian Blaga" University of Sibiu, Faculty of Sciences, Dr. Ion Raţiu Street 5-7, Sibiu, Romania, \\ RO-550012, angela.banaduc@ulbsibiu.ro, ad.banaduc@yahoo.com,dobre.alexandru95@yahoo.com \\ ** National Institute of Hydrology and Water Management, București-Ploiești Street 97, București, \\ Romania, RO-013686, rzvnvoicu@yahoo.com \\ *** Charles Stuart University, Institute for Land, Water and Society, P.O. Box 789, Albury, New South \\ Wales, Australia, AU-2640, lbaumgartner@csu.edu.au \\ **** Belgrade University, Studentski Street 16, Belgrade, Serbia, RS-11000, sasa@bio.bg.ac.rs
}

DOI: 10.2478/trser-2018-0021

KEYWORDS: Carpathian river, fragmentation mitigation technical elements.

\section{ABSTRACT}

The Bistra Mărului River fish fauna has been severely impacted by man-made activities, especially through longitudinal fragmentation, over the past 40 years. Fish fauna monitoring revealed structural changes and technical methods have been proposed, in order to restore the natural connectivity and the conservation of fish species. Benefits should accrue for key species: Salmo trutta fario, Cottus gobio, Thymallus thymallus, Eudontomyzon danfordi, Eudontomyzon vladykovi, Gobio uranoscopus, Barbus meridionalis, and Condrostoma nasus.

RÉSUMÉ: Solutions techniques pour atténuer les modifications des zones piscicoles touchées par la dégradation à long terme de l'habitat dans le Bistra Mărului - étude de cas.

La faune piscicole de la rivière Bistra Mărului a été soumise, durant les derniers 40 ans, à un fort impact anthropique, particulièrement due à la fragmentation longitudinale. La surveillance de l'ichtyofaune a mis en évidence le changement de sa structure. Des méthodes techniques ont été proposées, strictement adaptées aux catégories majeures de constructions hydrotechniques présentes sur la rivière étudiée, afin de remettre en état la connectivité naturelle et la conservation particulièrement des espèces clé de poissons: Salmo trutta fario, Cottus gobio, Thymallus thymallus, Eudontomyzon danfordi, Eudontomyzon vladykovi, Gobio uranoscopus, Barbus meridionalis et Condrostoma nasus.

REZUMAT: Soluții tehnice pentru reducerea deplasării zonelor ihtiologice afectate de degradarea habitatului pe termen lung în râul Bistra Mărului - studiu de caz.

Ihtiofauna râului Bistra Mărului a suferit în ultimii 40 de ani un impact antropic major, în special datorită fragmentării longitudinale. Monitoringul ihtiofaunei a relevat modificarea structurii acesteia. Au fost propuse metode tehnice adaptate strict la categoriile majore de construcții hidrotehnice prezente pe râul studiat, în vederea refacerii conectivităţii naturale și a conservării unor specii cheie de peşti: Salmo trutta fario, Cottus gobio, Thymallus thymallus, Eudontomyzon danfordi, Eudontomyzon vladykovi, Gobio uranoscopus, Barbus meridionalis și Condrostoma nasus. 


\section{INTRODUCTION}

Freshwater ecosystems are shared by multiple users and highly vulnerable to human interventions (Cambray and Bianco, 1998). Unfortunately, most systems are not inventoried sufficiently (Stiassny, 2002) due to the lack of resources. Freshwater systems, in this regard, have declined more rapidly than marine habitats (Sala et al., 2000). Streams and rivers are among the most degraded ecological systems in the world (Vörösmarty et al., 2010). Urgent steps are needed to come up with viable solutions that deal with development impacts.

The Danube Basin is the second largest, and most developed, in Europe. Extensive development over the past decades severely impacted aquatic habitats and fauna (Tockner et al., 2008; Bănăduc et al., 2016). A significant stressor which affects aquatic communities (Birk et al., 2012) is longitudinal fragmentation. The construction of regulators, dams and weirs can negatively influence the environmental river flow requirements (Acreman and Dunbar, 2004) and, subsequently, fish communities (Soolutayo, 2012; Olopade, 2013; Rumana et al., 2015). Fish are one of the most sensitive taxa group to reduced connectivity in Carpathian rivers and streams (Bănăduc 1999, 2000; Bănăduc et al., 2013; Voicu and Merten, 2014; Telcean and Cupşa, 2015; Voicu et al., 2017) because migration barriers can block access to important feeding, spawning and nursery habitat (Rathert et al., 1999; Oberdorff et al., 2011).

Globally, over 50,000 large dams and an unidentified number of smaller barriers for fish (Richter et al., 2010) threaten fish communities.

Barriers to upstream fish migration can be partially overcome by using fish passes; a channel through or around a migration barrier. If adapted to local specific conditions, even though the favorable outcome of such measures depends on multiple factors such as size of structure, volume and hydraulics of water moving through, fish communities can be rehabilitated (Mallen-Cooper and Brand, 2007). At structures like small culverts or weirs, solutions may be inexpensive (Katopodis, 1990; Aadland, 2010; Kemp and O’Hanley, 2010; Voicu et al., 2017, 2018). But it is important to note that fish passes need to be effective in a bi-directional sense. That is, consideration must be given to downstream movement to protect the downstream drift of fish eggs and larvae which is a characteristic of the life cycle of numerous fish species (Baumgartner et al., 2014).

In accordance with Water Framework Directive, river administrators must restore and maintain the longitudinal and lateral connectivity of rivers (Voicu and Merten, 2014). This is both a priority and a challenge for the specialists in the field (Voicu and Dominguez, 2016; Voicu and Baki, 2017). Restoring longitudinal connectivity is an essential component of the lotic ecosystems functions (Voicu and Breţcan, 2014) and must represent the basis of the protection of watercourses (Voicu et al., 2015). But rarely are potential impacts on fish considered in the design stages of river development programs.

We hypothesise that habitat fragmentation including habitat modifications, deficit and loss, is a long term driver of fish fauna change. A case study in the Timiş River basin (Fig. 1), Bistra Mărului River (Fig. 2) was implemented to determine the extent of human impacts and determine possible technical solutions.

Bistra Mărului River (Timiş and Danube basins) was selected for this study. The river has fish monitoring data from over 40 years and has many migration barriers, from the smallest $0.20 \mathrm{~cm}$ to the largest two m high. River flow is regulated by the Măru Dam of $125 \mathrm{~m}$ high (completed in 1992). 
The Timiş River basin is the main hydrographic basin of the south-western side of Romania, the largest basin from the historical region Banat, with a surface of $5,795 \mathrm{~km}^{2}$, its largest part laying on Romania's national territory. The upper side of the Timiş Basin includes the western slopes of the Banat Mountains, western slopes of the Tarcu Mountains, Muntele Mic Mountain and Poiana Ruscă Mountains and the Timiş-Cerna corridor. The middle-lower side of the Timiş Basin includes the following relief units: Lugoj Hills, Pogăniş Hills, Lugoj Plain and Timiş Plain. The extreme lower side of this basin is managed by Serbia.

Timiş Basin was significantly developed from the economic point of view since the 17th century. The current ecological state of some lotic sectors habitats has changed from "natural” to "severely modified"; and has significantly impacted fish populations. (Bănăduc et al., 2013; Burghelea et al., 2013; Stavrescu-Bedivan et al., 2017)

Comprehensive studies on the Timiș River ichthyofauna were made in the 60's of the last century (Bănărescu, 1964), and recently (Bănăduc et al., 2013).

Furthermore, it is known that species with high conservation value from the Bistra River to the Timiş River increases the conservative value and the resilience of the Timiş River sector from Maciova and Peștere towards downstream (Bănăduc et al., 2013).

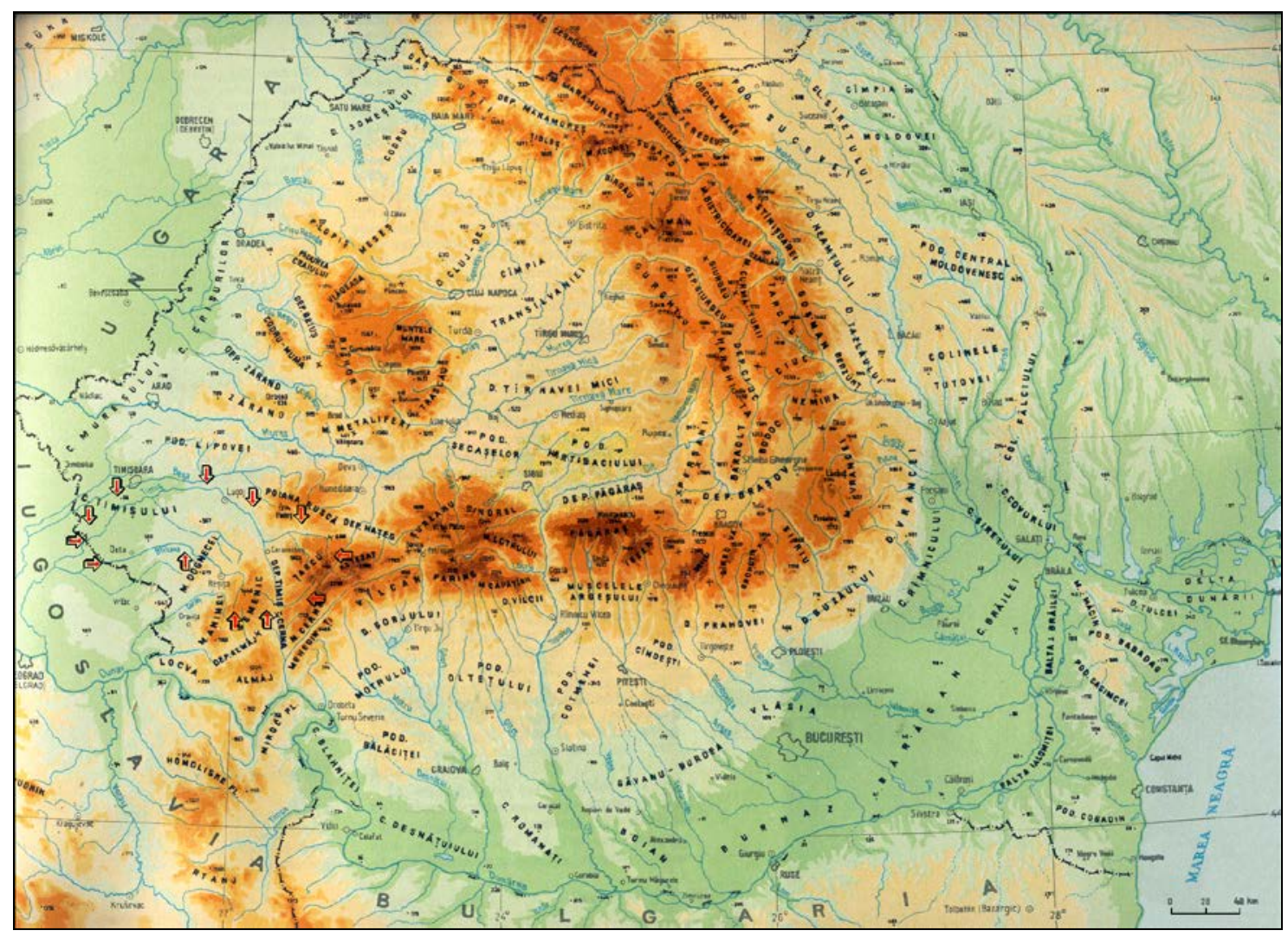

Figure 1: The Timiş River basin localization

(Badea et al., 1983 - modified). 


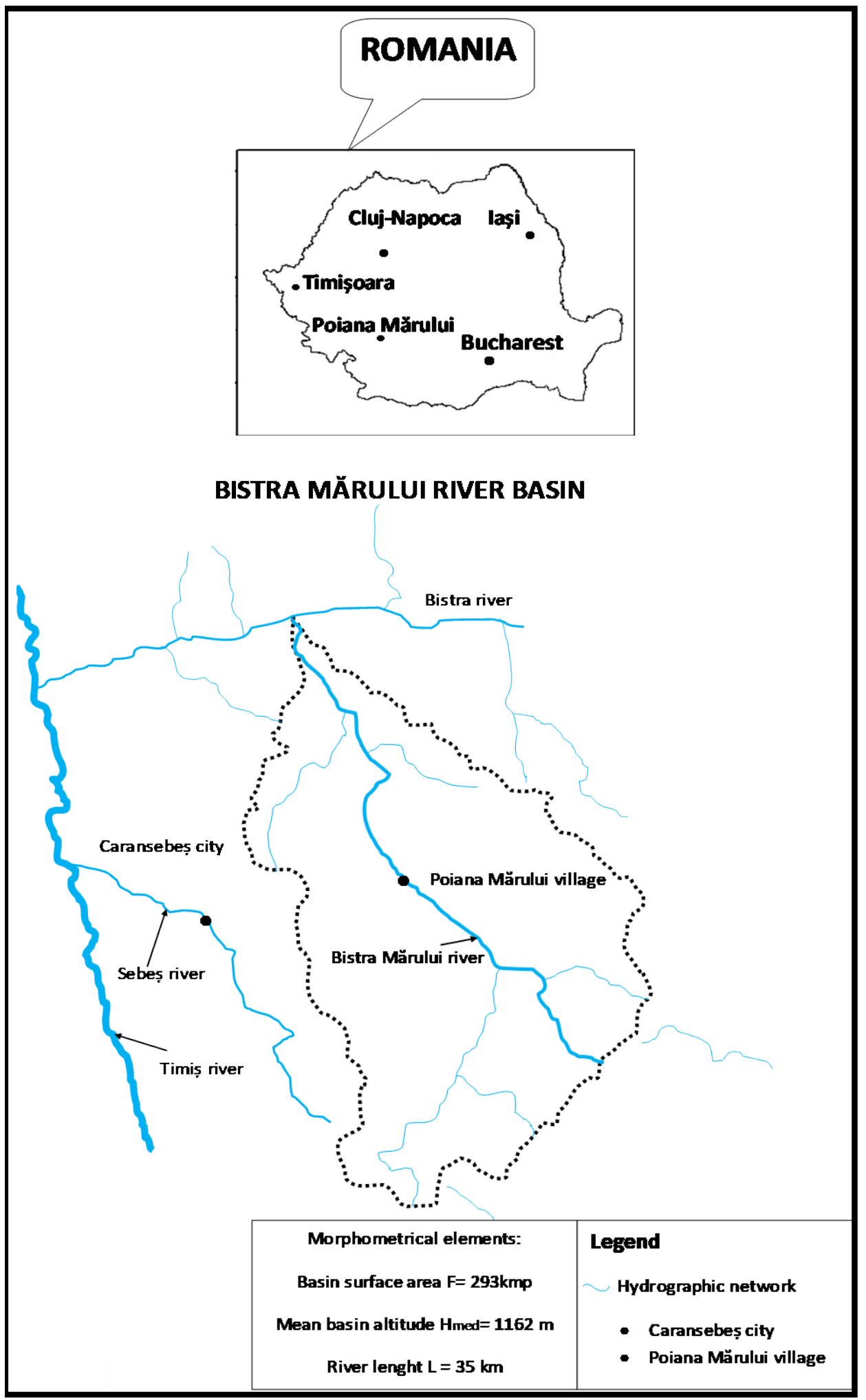

Figure 2: Bistra Mărului River basin. 
Environmental conditions and fish fauna vary both qualitatively and quantitatively in Carpathian streams, the habitat conditions variation led to the establishment of fish zonation based on the characteristic/indicator fish species (Bănărescu, 1964). For instance, large and medium size Carpathian rivers, which spring in mountain areas have five such specific fish zones: I brown trout zone, II grayling and Mediterannean barbell zone, III nase zone, IV barbell zone, and V carp zone. Zone (I) is characterised by a fairly constant and cold temperature (variations do not exceed $7-8^{\circ} \mathrm{C}$ maximum) fast flow, with waterfalls, oxygensaturated water, and short periods of frost. The indicator fish species of this zone is Salmo trutta fario, characteristic also being Cottus gobio, Phoxinus phoxinus, and Orthrias barbatulus.

This first section transitions into zone (II), which is dominated by fish species like: Barbus meridionalis, Thymallus thymallus, Alburnoides bipunctatus, Sabanejewia romanica, and Sabanejewia aurata. Zone (II) is characterised by a generally higher flow, less accentuated water current, generally devoid of waterfalls, permanently rocky bottom, being made up in general by of smaller boulders, water saturated in oxygen, oscillations of temperature $12-14^{\circ} \mathrm{C}$.

The final zone (III) includes the hilly lotic sectors, characterised in general by a hard rocky (sometimes mixed with pebbles, sand, clay and/or mud) riverbed substrata. The rivers' water temperature oscillations are relatively high $\left(18-19^{\circ} \mathrm{C}\right)$, in summer the water temperature can be over $20^{\circ} \mathrm{C}$. Water levels also fluctuate and are high in the periods with precipitations and snow melt. The water is often high turbidity and can freeze on long sectors in winter. Indicator species in this zone are Chondrostoma nasus, also numerous being Squalius cephalus, Barbus barbus, and Vimba vimba. Other fish species in this zone can be: Barbus meridionalis, Cottus gobio, Phoxinus phoxinus, Orthrias barbatulus, Gobio uranoscopus, Alburnoides bipunctatus, Gobio gobio, Gobio kessleri, Cobitis aurata, Cobitis romanica, and Alburnus alburnus.

\section{MATERIALS AND METHODS}

This ichthyological monitoring study was done on the whole $35 \mathrm{~km}$ length of Bistra Mărului River (at an average altitude of 1,162 m a.s.l.), a river with a basin of $293 \mathrm{~km}^{2}$, from its mountainous springs area to its end in the Oțelu Roşu locality, in the period 19802018. Among the total of 51 sampling stations (Fig. 3), 50 were lotic habitats stations with quantitative samples and one lenitic habitat (Poiana Mărului Lake) station with qualitative samples. For the stations localisation a "Garmin GPSmap 62s" global positioning system was used.

The fishing method which was used in this study, was the electrofishing in time/effort unit (30 minutes). The device which was used was an Aquatech IG 600, 30 A, $0.65 / 1.2 \mathrm{kw}$, with two net stopers. All the sampled fish, were identified, counted and immediately released in situ. The local fisherman captures were also checked, both in lenitic and lotic habitats.

The Carpathian Fish Index of Biotic Integrity (CF-IBI) (Bănăduc and Curtean-Bănăduc, 2002) was used in highlighting anthropogenic determined negative effects on fish fauna integrity, based on life history and feeding relationships selected metrics. 


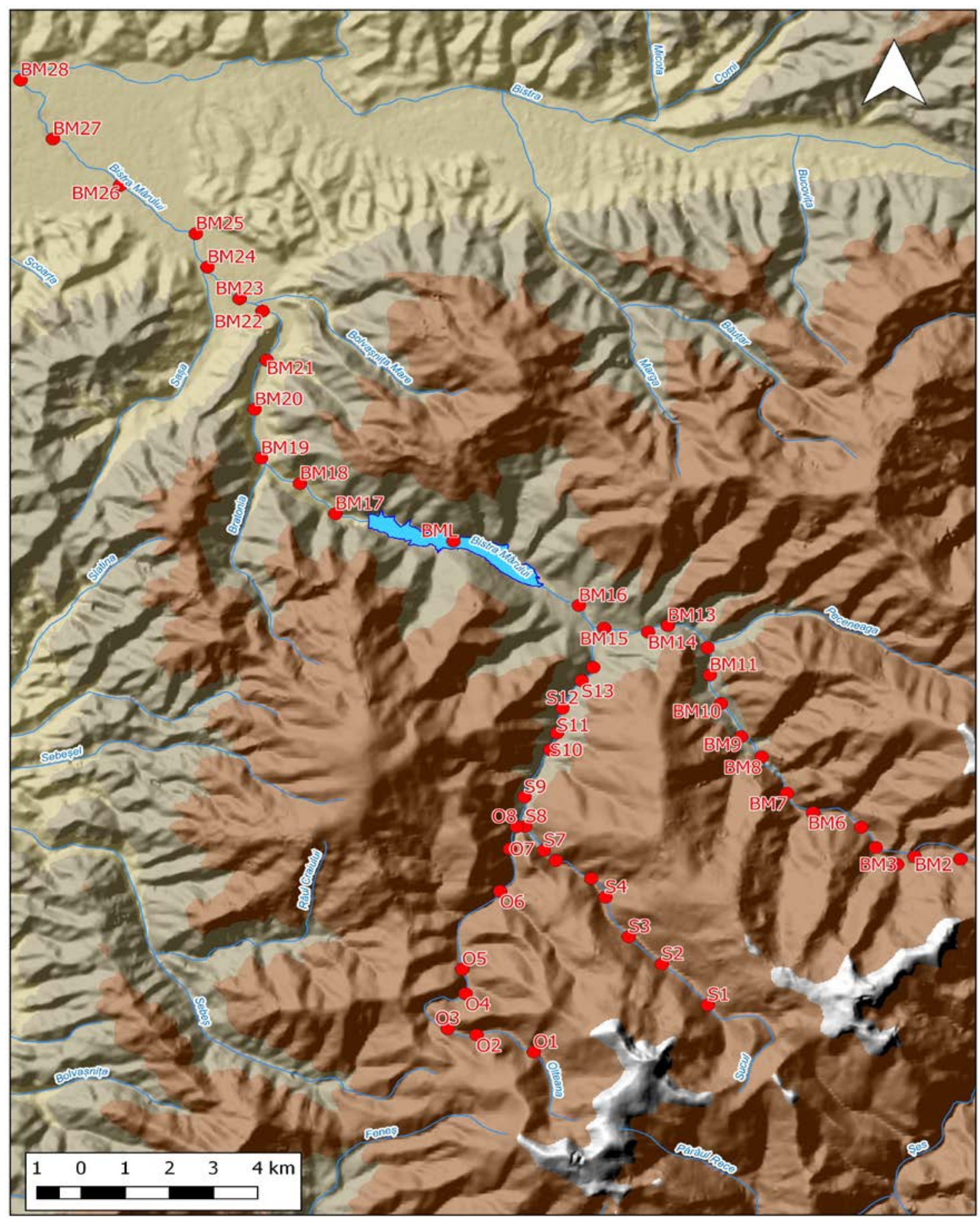

Figure 3: Sampling stations:

Bistra Mărului River $\left(\mathrm{BM}_{\mathrm{n}}\right)$, Şucu Stream $\left(\mathrm{S}_{\mathrm{n}}\right)$, Olteana Stream $\left(\mathrm{O}_{\mathrm{n}}\right)$, (GIS support - Pătrulescu A.). 


\section{RESULTS AND DISSCUSION}

This study is based on the long term monitoring results in Bistra Mărului River of the local fish fauna species and especialy on the folowings key and/or indicator fish species: Salmo trutta fario Linnaeus, 1758, Cottus gobio (Linnaeus, 1758), Thymallus thymallus (Linnaeus, 1758), Eudontomyzon danfordi Regan, 1911; Eudontomyzon vladykovi Oliva and Zanandrea, 1959, Gobio uranoscopus (Agassiz, 1828), Barbus meridionalis Riso, 1827, and Condrostoma nasus (Linnaeus, 1758).

The synthetic results of the Bistra Mărului River and two of its main tributaries (Şucu and Olteana streams) sampling stations (Fig. 3) fish fauna long term monitoring indicate a total catch of 19 fish species. The proportion of each species in the total catch changed substantially between 1980 and 2018 (Tabs. 1 and 2).

Table 1: The Şucu and Olteana streams, Bistra Mărului tributaries, sampling stations fish communities species; P/A - presence/absence, * parasite on Osteichthyes; ** in the substrate of the confluence areas of some small brooks tributaries of the Bistra Mărului Lake; CF-IBI values.

\begin{tabular}{|c|c|c|c|c|c|c|c|c|c|}
\hline Stations & Species & $\begin{array}{l}\dot{d} \\
\stackrel{\circ}{\circ} \\
\stackrel{9}{\sigma}\end{array}$ & 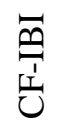 & 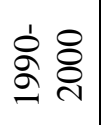 & 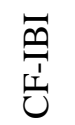 & 官 & 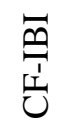 & 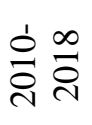 & $\underset{\substack{n \\
\mathcal{U}}}{\vec{n}}$ \\
\hline \multicolumn{10}{|c|}{ Şucu and Olteana streams - Bistra Mărului tributaries } \\
\hline$S_{1-4}$ & - & - & - & - & - & - & - & - & - \\
\hline $\mathrm{O}_{1-4}$ & - & - & - & - & - & - & - & - & - \\
\hline $\mathrm{S}_{5}$ & Salmo trutta fario & $\mathrm{P}$ & 45 & $\mathrm{P}$ & 45 & $\mathrm{P}$ & 45 & $\mathrm{P}$ & 45 \\
\hline $\mathrm{O}_{5}$ & - & - & - & - & - & - & - & - & - \\
\hline $\mathrm{S}_{6}$ & Salmo trutta fario & $\mathrm{P}$ & 45 & $\mathrm{P}$ & 45 & $\mathrm{P}$ & 45 & $\mathrm{P}$ & 45 \\
\hline $\mathrm{O}_{6}$ & Salmo trutta fario & $\mathrm{P}$ & 45 & $\mathrm{P}$ & 45 & $\mathrm{P}$ & 45 & $\mathrm{P}$ & 45 \\
\hline $\mathrm{S}_{7}$ & Salmo trutta fario & $\mathrm{P}$ & 45 & $\mathrm{P}$ & 45 & $\mathrm{P}$ & 45 & $\mathrm{P}$ & 45 \\
\hline $\mathrm{O}_{7}$ & Salmo trutta fario & $\mathrm{P}$ & 45 & $\mathrm{P}$ & 45 & $\mathrm{P}$ & 45 & $\mathrm{P}$ & 45 \\
\hline $\mathrm{S}_{8}$ & Salmo trutta fario & $\mathrm{P}$ & 45 & $\mathrm{P}$ & 45 & $\mathrm{P}$ & 45 & $\mathrm{P}$ & 45 \\
\hline $\mathrm{O}_{8}$ & Salmo trutta fario & $\mathrm{P}$ & 45 & $\mathrm{P}$ & 45 & $\mathrm{P}$ & 45 & $\mathrm{P}$ & 45 \\
\hline $\mathrm{S}_{9}$ & Salmo trutta fario & $\mathrm{P}$ & 45 & $\mathrm{P}$ & 45 & $\mathrm{P}$ & 45 & $\mathrm{P}$ & 45 \\
\hline $\mathrm{S}_{10}$ & Salmo trutta fario & $\mathrm{P}$ & 45 & $\mathrm{P}$ & 45 & $\mathrm{P}$ & 45 & $\mathrm{P}$ & 43 \\
\hline $\mathrm{S}_{11}$ & Salmo trutta fario & $\mathrm{P}$ & 45 & $\mathrm{P}$ & 45 & $\mathrm{P}$ & 45 & $\mathrm{P}$ & 43 \\
\hline \multirow{2}{*}{$\mathrm{S}_{12}$} & Salmo trutta fario & $\mathrm{P}$ & \multirow{2}{*}{45} & $\mathrm{P}$ & \multirow{2}{*}{45} & $\mathrm{P}$ & \multirow{2}{*}{45} & $\mathrm{P}$ & \multirow{2}{*}{43} \\
\hline & Cottus gobio & $\mathrm{P}$ & & $\mathrm{P}$ & & $\mathrm{P}$ & & $\mathrm{P}$ & \\
\hline \multirow{2}{*}{$\mathrm{S}_{13}$} & Salmo trutta fario & $\mathrm{P}$ & \multirow{2}{*}{45} & $\mathrm{P}$ & \multirow{2}{*}{45} & $\mathrm{P}$ & \multirow{2}{*}{45} & $\mathrm{P}$ & \multirow{2}{*}{43} \\
\hline & Cottus gobio & $\mathrm{P}$ & & $\mathrm{P}$ & & $\mathrm{P}$ & & $\mathrm{P}$ & \\
\hline \multirow{3}{*}{$\mathrm{S}_{14}$} & Salmo trutta fario & $\mathrm{P}$ & \multirow{3}{*}{45} & $\mathrm{P}$ & \multirow{3}{*}{45} & $\mathrm{P}$ & \multirow{3}{*}{45} & $\mathrm{P}$ & \multirow{3}{*}{43} \\
\hline & Cottus gobio & $P$ & & $\mathrm{P}$ & & $\mathrm{P}$ & & $\mathrm{P}$ & \\
\hline & Eudontomyzon danfordi & $\mathrm{P}$ & & $\mathrm{P}$ & & $\mathrm{P}$ & & $\mathrm{P}$ & \\
\hline
\end{tabular}


Table 2: The Bistra Mărului River sampling stations fish communities species; P/A presence/absence, * parasite on Osteichthyes; ${ }^{* *}$ in the substrate of the confluence areas of some small brooks tributaries of the Bistra Mărului Lake; CF-IBI values.

\begin{tabular}{|c|c|c|c|c|c|c|c|c|c|}
\hline \multicolumn{10}{|c|}{ Bistra Mărului River } \\
\hline $\mathrm{BM}_{1-3}$ & - & - & - & - & - & - & - & - & - \\
\hline $\mathrm{BM}_{4-9}$ & Salmo trutta fario & $\mathrm{P}$ & 45 & $\mathrm{P}$ & 45 & $\mathrm{P}$ & 44 & $\mathrm{P}$ & 43 \\
\hline \multirow{2}{*}{$\mathrm{BM}_{10}$} & Salmo trutta fario & $\mathrm{P}$ & \multirow{2}{*}{45} & $\mathrm{P}$ & \multirow{2}{*}{43} & $\mathrm{P}$ & \multirow{2}{*}{43} & $\mathrm{P}$ & \multirow{2}{*}{43} \\
\hline & Cottus gobio & $\mathrm{P}$ & & $\mathrm{P}$ & & $\mathrm{P}$ & & $\mathrm{P}$ & \\
\hline \multirow{2}{*}{$\mathrm{BM}_{11}$} & Salmo trutta fario & $\mathrm{P}$ & \multirow{2}{*}{45} & $\mathrm{P}$ & \multirow{2}{*}{43} & $\mathrm{P}$ & \multirow{2}{*}{43} & $\mathrm{P}$ & \multirow{2}{*}{43} \\
\hline & Cottus gobio & $\mathrm{P}$ & & $\mathrm{P}$ & & $\mathrm{P}$ & & $\mathrm{P}$ & \\
\hline \multirow{3}{*}{$\mathrm{BM}_{12}$} & Salmo trutta fario & $\mathrm{P}$ & \multirow{3}{*}{45} & $\mathrm{P}$ & \multirow{3}{*}{44} & $\mathrm{P}$ & \multirow{3}{*}{44} & $\mathrm{P}$ & \multirow{3}{*}{42} \\
\hline & Cottus gobio & $\mathrm{P}$ & & $\mathrm{P}$ & & $\mathrm{P}$ & & $\mathrm{P}$ & \\
\hline & Eudontomyzon danfordi & $\mathrm{P}$ & & $\mathrm{P}$ & & $\mathrm{P}$ & & $\mathrm{P}$ & \\
\hline \multirow{3}{*}{$\mathrm{BM}_{13}$} & Salmo trutta fario & $\mathrm{P}$ & \multirow{3}{*}{45} & $\mathrm{P}$ & \multirow{3}{*}{44} & $\mathrm{P}$ & \multirow{3}{*}{44} & $\mathrm{P}$ & \multirow{3}{*}{42} \\
\hline & Cottus gobio & $\mathrm{P}$ & & $\mathrm{P}$ & & $\mathrm{P}$ & & $\mathrm{P}$ & \\
\hline & Eudontomyzon danfordi & $\mathrm{P}$ & & $\mathrm{P}$ & & $\mathrm{P}$ & & $\mathrm{P}$ & \\
\hline \multirow{3}{*}{$\mathrm{BM}_{13}$} & Salmo trutta fario & $\mathrm{P}$ & \multirow{3}{*}{45} & $\mathrm{P}$ & \multirow{3}{*}{44} & $\mathrm{P}$ & \multirow{3}{*}{44} & $\mathrm{P}$ & \\
\hline & Cottus gobio & $\mathrm{P}$ & & $\mathrm{P}$ & & $\mathrm{P}$ & & $\mathrm{P}$ & 42 \\
\hline & Eudontomyzon danfordi & $\mathrm{P}$ & & $\mathrm{P}$ & & $\mathrm{P}$ & & $\mathrm{P}$ & \\
\hline & Salmo trutta fario & $\mathrm{P}$ & & $\mathrm{P}$ & & $\mathrm{P}$ & & $\mathrm{P}$ & \\
\hline $\mathrm{BM}_{14}$ & Cottus gobio & $\mathrm{P}$ & 45 & $\mathrm{P}$ & 44 & $\mathrm{P}$ & 44 & $\mathrm{P}$ & 42 \\
\hline & Eudontomyzon danfordi & $\mathrm{P}$ & & $\mathrm{P}$ & & $\mathrm{P}$ & & $\mathrm{P}$ & \\
\hline & Salmo trutta fario & $\mathrm{P}$ & & $\mathrm{P}$ & & $\mathrm{P}$ & & $\mathrm{P}$ & \\
\hline & Cottus gobio & $\mathrm{P}$ & & A & & A & & A & \\
\hline & Eudontomyzon danfordi & $\mathrm{P}$ & & $\mathrm{A}$ & & $\mathrm{P}$ & & $\mathrm{P}$ & \\
\hline $\mathrm{BM}_{15}$ & Phoxinus phoxinus & A & 45 & A & 42 & $P$ & 35 & $\mathrm{P}$ & 30 \\
\hline & Sabanejewia aurata $b$. & A & & A & & $\mathrm{P}$ & & $\begin{array}{l}P \\
P\end{array}$ & \\
\hline & Alburnoides bipunctatus & A & & A & & A & & $\begin{array}{l}\mathrm{r} \\
\mathrm{P}\end{array}$ & \\
\hline & Squalius cephalus & $\mathrm{P}$ & & A & & $\mathrm{P}$ & & $\mathrm{P}$ & \\
\hline & Salmo trutta fario & $\mathrm{P}$ & & $\mathrm{P}$ & & $\mathrm{P}$ & & $\mathrm{P}$ & \\
\hline & Cottus gobio & $\mathrm{P}$ & & $\mathrm{P}$ & & $\mathrm{P}$ & & $\mathrm{P}$ & \\
\hline & Eudontomyzon danfordi* & $\mathrm{P}$ & & $\mathrm{P}$ & & $\mathrm{P}$ & & $\mathrm{P}$ & \\
\hline & Eudontomyzon vladykovi** & $\mathrm{P}$ & & $\mathrm{P}$ & & $\mathrm{P}$ & & $\mathrm{P}$ & \\
\hline & Phoxinus phoxinus & A & & $\mathrm{P}$ & & $\mathrm{P}$ & & $\mathrm{P}$ & \\
\hline & Sabanejewia aurata $b$. & $\mathrm{A}$ & & $\mathrm{P}$ & & $\mathrm{P}$ & & $\mathrm{P}$ & \\
\hline BML & Alburnoides bipunctatus & $\mathrm{A}$ & 45 & $\mathrm{P}$ & 35 & $\mathrm{P}$ & 25 & $\mathrm{P}$ & 15 \\
\hline & Alburnus alburnus & A & & A & & $\mathrm{P}$ & & $\mathrm{P}$ & \\
\hline & Squalius cephalus & A & & $\mathrm{A}$ & & $\mathrm{P}$ & & $\mathrm{P}$ & \\
\hline & $\begin{array}{l}\text { Scardinius } \\
\text { erythrophthalmus }\end{array}$ & A & & A & & A & & $\mathrm{P}$ & \\
\hline & Carassius gibelio & A & & A & & A & & $\mathrm{P}$ & \\
\hline & Esox lucius & A & & A & & A & & $\mathrm{P}$ & \\
\hline
\end{tabular}


Table 2 (continued): The Bistra Mărului River sampling stations fish communities species; P/A - presence/absence, ${ }^{*}$ parasite on Osteichthyes; ${ }^{* *}$ in the substrate of the confluence areas of some small brooks tributaries of the Bistra Mărului Lake; CF-IBI values.

\begin{tabular}{|c|c|c|c|c|c|c|c|c|c|}
\hline Stations & Species & 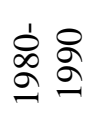 & 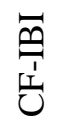 & 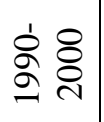 & 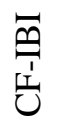 & 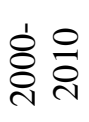 & 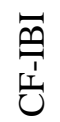 & 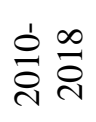 & 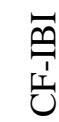 \\
\hline \multicolumn{10}{|c|}{ Bistra Mărului River } \\
\hline \multirow{4}{*}{$\mathrm{BM}_{16}$} & Salmo trutta fario & $\mathrm{P}$ & \multirow{4}{*}{45} & A & \multirow{4}{*}{1} & $\mathrm{P}$ & \multirow{4}{*}{36} & $\mathrm{P}$ & \multirow{4}{*}{36} \\
\hline & Cottus gobio & $\mathrm{P}$ & & A & & A & & A & \\
\hline & Eudontomyzon danfordi & $\mathrm{P}$ & & A & & A & & A & \\
\hline & Thymallus thymallus & $\mathrm{P}$ & & A & & A & & A & \\
\hline \multirow{4}{*}{$\mathrm{BM}_{17}$} & Salmo trutta fario & $\mathrm{P}$ & \multirow{4}{*}{45} & $\mathrm{P}$ & \multirow{4}{*}{36} & $\mathrm{P}$ & \multirow{4}{*}{31} & $\mathrm{P}$ & \multirow{4}{*}{30} \\
\hline & Cottus gobio & $\mathrm{P}$ & & $\mathrm{P}$ & & $\mathrm{P}$ & & A & \\
\hline & Eudontomyzon danfordi & $\mathrm{P}$ & & $\mathrm{P}$ & & A & & A & \\
\hline & Thymallus thymallus & $\mathrm{P}$ & & A & & A & & A & \\
\hline \multirow{4}{*}{$\mathrm{BM}_{18}$} & Salmo trutta fario & $\mathrm{P}$ & \multirow{4}{*}{45} & $\mathrm{P}$ & \multirow{4}{*}{36} & $\mathrm{P}$ & \multirow{4}{*}{42} & $\mathrm{P}$ & \multirow{4}{*}{30} \\
\hline & Cottus gobio & $\mathrm{P}$ & & $\mathrm{P}$ & & $\mathrm{P}$ & & A & \\
\hline & Eudontomyzon danfordi & $\mathrm{P}$ & & $\mathrm{P}$ & & $\mathrm{P}$ & & $\mathrm{P}$ & \\
\hline & Thymallus thymallus & $\mathrm{P}$ & & A & & A & & A & \\
\hline \multirow{4}{*}{$\mathrm{BM}_{19}$} & Salmo trutta fario & $\mathrm{P}$ & \multirow{4}{*}{45} & $\mathrm{P}$ & \multirow{4}{*}{36} & $\mathrm{P}$ & \multirow{4}{*}{42} & $\mathrm{P}$ & \multirow{4}{*}{30} \\
\hline & Cottus gobio & $\mathrm{P}$ & & $\mathrm{P}$ & & $\mathrm{P}$ & & A & \\
\hline & Eudontomyzon danfordi & $\mathrm{P}$ & & $\mathrm{P}$ & & $\mathrm{P}$ & & $\mathrm{P}$ & \\
\hline & Thymallus thymallus & $\mathrm{P}$ & & A & & A & & A & \\
\hline \multirow{4}{*}{$\mathrm{BM}_{20}$} & Salmo trutta fario & $\mathrm{P}$ & \multirow{4}{*}{45} & $\mathrm{P}$ & \multirow{4}{*}{36} & $\mathrm{P}$ & \multirow{4}{*}{42} & $\mathrm{P}$ & \multirow{4}{*}{30} \\
\hline & Cottus gobio & $\mathrm{P}$ & & $\mathrm{P}$ & & $\mathrm{P}$ & & A & \\
\hline & Eudontomyzon danfordi & $\mathrm{P}$ & & $\mathrm{P}$ & & $\mathrm{P}$ & & $\mathrm{P}$ & \\
\hline & Thymallus thymallus & $\mathrm{P}$ & & A & & A & & A & \\
\hline \multirow{4}{*}{$\mathrm{BM}_{21}$} & Salmo trutta fario & $\mathrm{P}$ & \multirow{4}{*}{45} & $\mathrm{P}$ & \multirow{4}{*}{36} & $\mathrm{P}$ & & $\mathrm{P}$ & \\
\hline & Cottus gobio & $\mathrm{P}$ & & $\mathrm{P}$ & & $\mathrm{P}$ & & A & \\
\hline & Eudontomyzon danfordi & $\mathrm{P}$ & & $\mathrm{P}$ & & $\mathrm{P}$ & 42 & $\mathrm{P}$ & 30 \\
\hline & Thymallus thymallus & $\mathrm{P}$ & & A & & A & & A & \\
\hline & Salmo trutta fario & $\mathrm{P}$ & & $\mathrm{P}$ & & $\mathrm{P}$ & & $\mathrm{P}$ & \\
\hline $\mathrm{BM}_{2}$ & Cottus gobio & $\mathrm{P}$ & 45 & $\mathrm{P}$ & 36 & $\mathrm{P}$ & 42 & A & 30 \\
\hline $\mathrm{BIM}_{22}$ & Eudontomyzon danfordi & $\mathrm{P}$ & 45 & $\mathrm{P}$ & 30 & $\mathrm{P}$ & 42 & $\mathrm{P}$ & 30 \\
\hline & Thymallus thymallus & $\mathrm{P}$ & & $\mathrm{A}$ & & $\mathrm{A}$ & & A & \\
\hline
\end{tabular}


Table 2 (continued): The Bistra Mărului River sampling stations fish communities species; P/A - presence/absence, * parasite on Osteichthyes; ** in the substrate of the confluence areas of some small brooks tributaries of the Bistra Mărului Lake; CF-IBI values.

\begin{tabular}{|c|c|c|c|c|c|c|c|c|c|}
\hline Stations & Species & $\begin{array}{l}\stackrel{8}{\circ} \\
\stackrel{2}{\sigma}\end{array}$ & $\underset{\overrightarrow{\mid c}}{\overline{\mid c}}$ & 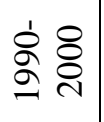 & 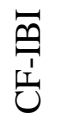 & 客 & 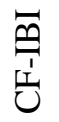 & 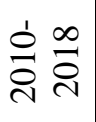 & 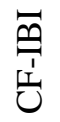 \\
\hline \multicolumn{10}{|c|}{ Bistra Mărului River } \\
\hline \multirow{10}{*}{$\mathrm{BM}_{23}$} & Salmo trutta fario & $\mathrm{P}$ & \multirow{10}{*}{45} & $\mathrm{P}$ & \multirow{10}{*}{36} & $\mathrm{P}$ & \multirow{10}{*}{34} & A & \multirow{10}{*}{24} \\
\hline & Cottus gobio & $\mathrm{P}$ & & $\mathrm{P}$ & & A & & A & \\
\hline & Eudontomyzon danfordi & $\mathrm{P}$ & & $\mathrm{P}$ & & $\mathrm{P}$ & & A & \\
\hline & Thymallus thymallus & $\mathrm{P}$ & & $\mathrm{P}$ & & A & & A & \\
\hline & Phoxinus phoxinus & A & & $\mathrm{P}$ & & $\mathrm{P}$ & & $\mathrm{P}$ & \\
\hline & Sabanejewia a. balcanica & A & & $\mathrm{P}$ & & $\mathrm{P}$ & & $\mathrm{P}$ & \\
\hline & Barbus meridionalis & $\mathrm{A}$ & & $\mathrm{A}$ & & $\mathrm{A}$ & & $\mathrm{P}$ & \\
\hline & Squalius cephalus & A & & $\mathrm{A}$ & & $\mathrm{A}$ & & $\mathrm{P}$ & \\
\hline & Orthrias barbatulus & $\mathrm{A}$ & & $\mathrm{P}$ & & $\mathrm{P}$ & & $\mathrm{P}$ & \\
\hline & Alburnoides bipunctatus & $\mathrm{A}$ & & $\mathrm{A}$ & & $\mathrm{P}$ & & $\mathrm{P}$ & \\
\hline \multirow{10}{*}{$\mathrm{BM}_{24}$} & Salmo trutta fario & $\mathrm{P}$ & \multirow{10}{*}{45} & $\mathrm{P}$ & \multirow{10}{*}{36} & $\mathrm{P}$ & \multirow{10}{*}{34} & A & \multirow{10}{*}{24} \\
\hline & Cottus gobio & $\mathrm{P}$ & & $\mathrm{P}$ & & A & & A & \\
\hline & Eudontomyzon danfordi & $\mathrm{P}$ & & $\mathrm{P}$ & & $\mathrm{P}$ & & $\mathrm{A}$ & \\
\hline & Thymallus thymallus & $\mathrm{P}$ & & $\mathrm{P}$ & & A & & A & \\
\hline & Phoxinus phoxinus & A & & $\mathrm{P}$ & & $\mathrm{P}$ & & $\mathrm{P}$ & \\
\hline & Sabanejewia a. balcanica & A & & $\mathrm{P}$ & & $\mathrm{P}$ & & $\mathrm{P}$ & \\
\hline & Barbus meridionalis & A & & A & & A & & $\mathrm{P}$ & \\
\hline & Squalius cephalus & A & & A & & A & & $\mathrm{P}$ & \\
\hline & Orthrias barbatulus & A & & $\mathrm{P}$ & & $\mathrm{P}$ & & $\mathrm{P}$ & \\
\hline & Alburnoides bipunctatus & A & & A & & $\mathrm{P}$ & & $\mathrm{P}$ & \\
\hline \multirow{13}{*}{$\mathrm{BM}_{25}$} & Salmo trutta fario & A & \multirow{13}{*}{42} & A & \multirow{13}{*}{36} & A & \multirow{13}{*}{29} & A & \multirow{13}{*}{22} \\
\hline & Cottus gobio & A & & A & & A & & A & \\
\hline & Eudontomyzon danfordi & A & & A & & A & & A & \\
\hline & Thymallus thymallus & $\mathrm{P}$ & & $\mathrm{P}$ & & A & & A & \\
\hline & Phoxinus phoxinus & $\mathrm{P}$ & & $\mathrm{P}$ & & $\mathrm{P}$ & & $\mathrm{P}$ & \\
\hline & Sabanejewia a. balcanica & $\mathrm{A}$ & & $\mathrm{P}$ & & $\mathrm{P}$ & & $\mathrm{P}$ & \\
\hline & Barbus meridionalis & A & & A & & $\mathrm{P}$ & & $\mathrm{P}$ & \\
\hline & Squalius cephalus & A & & A & & A & & $\mathrm{P}$ & \\
\hline & Gobio gobio & A & & A & & $\mathrm{P}$ & & $\mathrm{P}$ & \\
\hline & Orthrias barbatulus & $\mathrm{P}$ & & $\mathrm{P}$ & & $\mathrm{P}$ & & $\mathrm{P}$ & \\
\hline & Alburnoides bipunctatus & $\mathrm{P}$ & & $\mathrm{P}$ & & $\mathrm{P}$ & & $\mathrm{P}$ & \\
\hline & Gobio uranoscopus & $\mathrm{P}$ & & $\mathrm{P}$ & & $\mathrm{A}$ & & $\mathrm{A}$ & \\
\hline & Chondrostoma nasus & $\mathrm{P}$ & & $\mathrm{P}$ & & $\mathrm{P}$ & & A & \\
\hline
\end{tabular}


Table 2 (continued): The Bistra Mărului River sampling stations fish communities species; P/A - presence/absence, * parasite on Osteichthyes; ** in the substrate of the confluence areas of some small brooks tributaries of the Bistra Mărului Lake; CF-IBI values.

\begin{tabular}{|c|c|c|c|c|c|c|c|c|c|}
\hline Stations & Species & 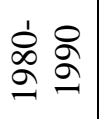 & 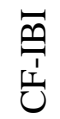 & 官 \& & 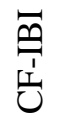 & 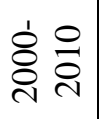 & $\underset{\vec{t}}{\vec{T}}$ & 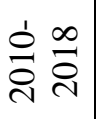 & $\underset{\vec{c}}{\overrightarrow{\underline{T}}}$ \\
\hline \multicolumn{10}{|c|}{ Bistra Mărului River } \\
\hline \multirow{13}{*}{$\mathrm{BM}_{26}$} & Salmo trutta fario & A & \multirow{13}{*}{42} & A & \multirow{13}{*}{36} & A & \multirow{13}{*}{29} & A & \multirow{13}{*}{22} \\
\hline & Cottus gobio & A & & A & & A & & A & \\
\hline & Eudontomyzon danfordi & A & & A & & A & & A & \\
\hline & Thymallus thymallus & $\mathrm{P}$ & & $\mathrm{P}$ & & A & & A & \\
\hline & Phoxinus phoxinus & $\mathrm{P}$ & & $\mathrm{P}$ & & $\mathrm{P}$ & & $\mathrm{P}$ & \\
\hline & Sabanejewia a. balcanica & A & & $\mathrm{P}$ & & $\mathrm{P}$ & & $\mathrm{P}$ & \\
\hline & Barbus meridionalis & A & & A & & $\mathrm{P}$ & & $\mathrm{P}$ & \\
\hline & Squalius cephalus & $\mathrm{A}$ & & $\mathrm{A}$ & & $\mathrm{A}$ & & $\mathrm{P}$ & \\
\hline & Gobio gobio & A & & A & & $\mathrm{P}$ & & $\mathrm{P}$ & \\
\hline & Orthrias barbatulus & $\mathrm{P}$ & & $\mathrm{P}$ & & $\mathrm{P}$ & & $\mathrm{P}$ & \\
\hline & Alburnoides bipunctatus & $\mathrm{P}$ & & $\mathrm{P}$ & & $\mathrm{P}$ & & $\mathrm{P}$ & \\
\hline & Gobio uranoscopus & $\mathrm{P}$ & & $\mathrm{P}$ & & A & & A & \\
\hline & Chondrostoma nasus & $\mathrm{P}$ & & $\mathrm{P}$ & & $\mathrm{A}$ & & $\mathrm{A}$ & \\
\hline \multirow{13}{*}{$\mathrm{BM}_{28}$} & Salmo trutta fario & A & \multirow{13}{*}{42} & A & \multirow{13}{*}{36} & A & \multirow{13}{*}{29} & A & \multirow{13}{*}{22} \\
\hline & Cottus gobio & A & & A & & $\mathrm{A}$ & & A & \\
\hline & Eudontomyzon danfordi & $\mathrm{A}$ & & $\mathrm{A}$ & & $\mathrm{A}$ & & $\mathrm{A}$ & \\
\hline & Thymallus thymallus & $\mathrm{P}$ & & $\mathrm{P}$ & & $\mathrm{A}$ & & $\mathrm{A}$ & \\
\hline & Phoxinus phoxinus & $\mathrm{P}$ & & $\mathrm{P}$ & & $\mathrm{P}$ & & $\mathrm{P}$ & \\
\hline & Sabanejewia a. balcanica & A & & $\mathrm{P}$ & & $\mathrm{P}$ & & $\mathrm{P}$ & \\
\hline & Barbus meridionalis & $\mathrm{A}$ & & $\mathrm{A}$ & & $\mathrm{P}$ & & $\mathrm{P}$ & \\
\hline & Squalius cephalus & $\mathrm{A}$ & & $\mathrm{A}$ & & $\mathrm{A}$ & & $\mathrm{P}$ & \\
\hline & Gobio gobio & A & & A & & $\mathrm{P}$ & & $\mathrm{P}$ & \\
\hline & Orthrias barbatulus & $\mathrm{P}$ & & $\mathrm{P}$ & & $\mathrm{P}$ & & $\mathrm{P}$ & \\
\hline & Alburnoides bipunctatus & $\mathrm{P}$ & & $\mathrm{P}$ & & $\mathrm{P}$ & & $\mathrm{P}$ & \\
\hline & Gobio uranoscopus & $\mathrm{P}$ & & $\mathrm{P}$ & & A & & A & \\
\hline & Chondrostoma nasus & $\mathrm{P}$ & & $\mathrm{P}$ & & A & & $\mathrm{A}$ & \\
\hline
\end{tabular}




\section{trend}

Key species, common not sensible species, ichthyological zones evolution and

Salmo trutta fario Linnaeus, 1758; Brown trout; Order Salmoniformes, Family Salmonidae. The decreasing abundance and spread with arround $50 \%$, especialy in the middle and lower sectors, in the studied decades in Bistra Mărului basin, reflect the human activities (mainly damming, but also poaching, pollution and riverbed exploitation) negative impact on river continuum, water flowing regime and temperature and oxygenation. In the present the trend of this species was consider as a decreasing one, till the moment when a proper management measures will be designed and constantly implemented in the field.

Cottus gobio (Linnaeus, 1758); Miller's thumb, Sculpin, Bullhead; Order Scorpaeniformes, Family Cottidae; Natura 2000 Code - 1163. This protected species is in a worse situation than the brown trout, decreasing abundance and spread with over $75 \%$, due to the same anthropogenicaly reasons at which have to be add the fact that no stocking and restoking are made like for Salmo trutta fario and their direct competition (these two species adults eat the juveniles of the other one) and indirect trophic competition (relatively simmilar trophic macroinvertebrate base at least for some age classes individuals).

Thymallus thymallus (Linnaeus, 1758); European grayling; Order Salmoniformes, Family Salmonidae, Natura 2000 Code - 1109. The 100\% disappearance of this fish species in the studied river is largely due to fragmentation. These speices are still present in the near Bistra, Mara and Bistra Bouţarului rivers, where a well balanced population of grayling still thrive.

Eudontomyzon danfordi Regan, 1911 (Bănărescu, 1969, 2005); Carpathian lamprey; Order Petromyzontiformes, Family Petromyzontidae; Natura 2000 Code -4123 . Decreased by $50 \%$ but is not protected, especially in the middle and lower reaches. Fragmentation and habitat isoltation is likely a large impact. It is interesting to highlight that in its lower confluence area and its near Mara, Bistra Bouţarului and Bistra rivers are characterised by stable populations of Eudontomyzon danfordi.

Eudontomyzon vladykovi Oliva and Zanandrea, 1959; Danubian brook lamprey; Natura 2000 Code - 4123; Order Petromyzontiformes, Family Petromyzontidae. This elusive extremely rare protected species in the Romanian Carpathians it is unfortunately on the brink of local extinction, due to the fact that its small sector of existence was inundated by the Bistra Mărului big lake. In the last decade it was found a single individual in the stomach of a Squalius cephalus captured in the lake, that individual caming from one of the local small tributaries of this anthropogenic lake.

Gobio uranoscopus (Agassiz, 1828); Danube Gudgeon; Order Cypriniformes; Family Cyprinidae; Natura 2000 Code - 1122. Is now locally extinct and significantly impacted by migratory obstruction. Good populations of this species appear again in the lower Bistra River.

Barbus meridionalis Riso, 1827; Mediterranean barbell; Order Cypriniformes; Family Cyprinidae; Natura 2000 Code - 1138. It is a protected species which after the lotic habitat conditions in the middle and lower sectors changed due to the human impact, collonised from downstream and replaced some of the more upper described protected fish species.

Chondrostoma nasus (Linnaeus, 1758); Common nase; Order Cypriniformes; Family Cyprinidae. This key species has been absent for over a decade, in spite of the fact that it is still present downstream in Bistra River. 
As a whole, the fish comunities ecological status analysed based on the CF-IBI (Tabs. 1 and 2) register drastic deacrising especialy in the middle and lower streches and in the periods 1990-2000 - 2000-2010 - 2010-2018 (Fig. 4).

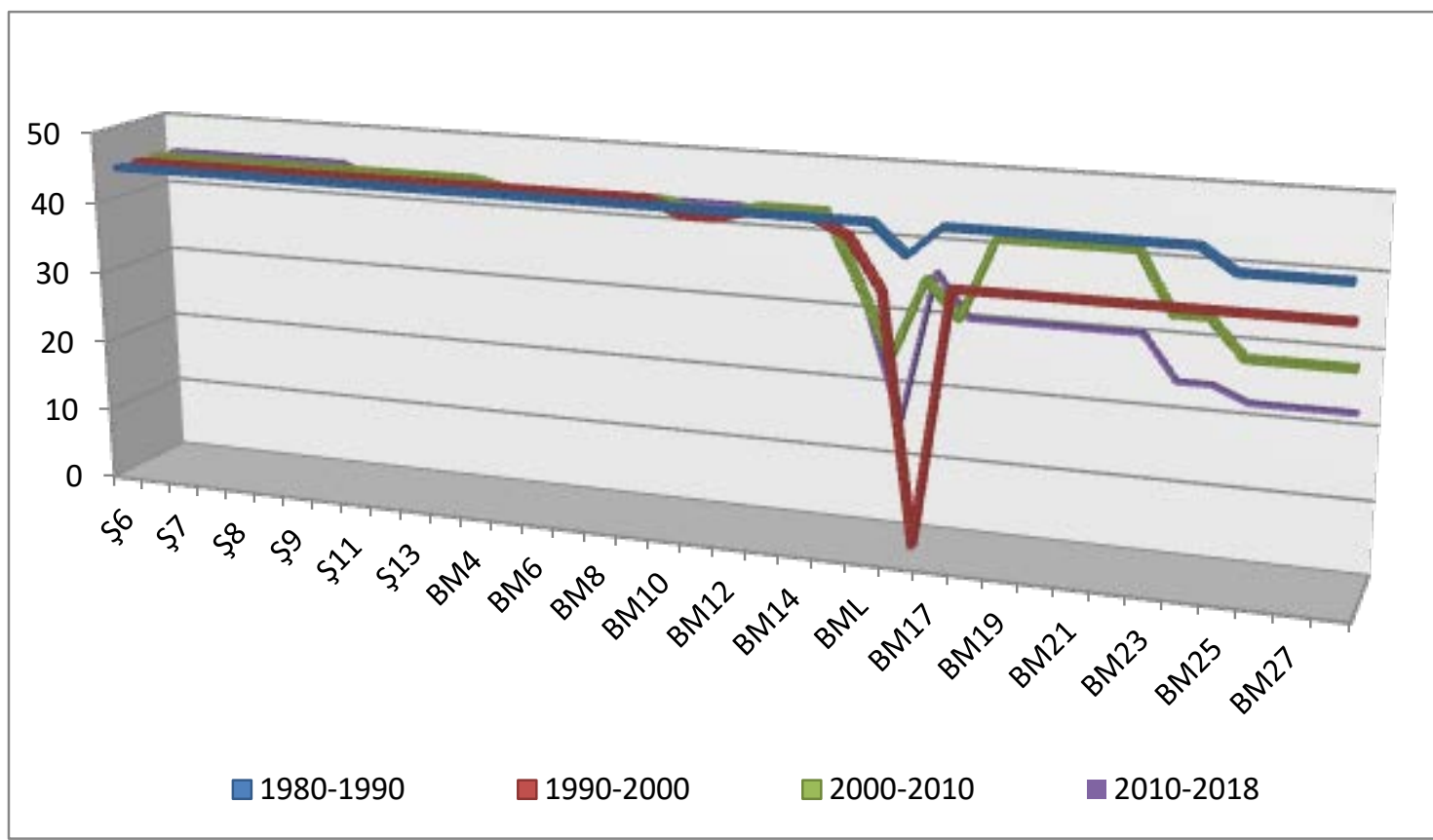

Figure 4: The decreasing trend of CF-IBI values/ecological status along the Bistra Mărului River and its tributaries Şucu and Olteana streams.

\section{Technical proposals for diminishing the human impact of lotic fragmentation}

1. The upper Bistra Mărului River and its tributaries Şucu and Olteana (50 sampling stations) has over 20 variable in size (small to medium: $0.20 \mathrm{~cm}-2 \mathrm{~m}$ ) human obstacles, which can be split in two main categories, which need two main categories of technical proposals with the needed role of reconect the longitudinal lotic sectors for the improoving the local fish fauna.

1.1 Spillways first category of proposed fish migration system solution - discharge sill located on a Bistra Mărului tributary

On a tributary of the Bistra River (even at the confluence with it) (Fig. 4a) there is a spill threshold (1) of about $15 \mathrm{~m}$ long, the width of the canopy of about one $\mathrm{m}$ and the height of $2.3 \mathrm{~m}$ (Fig. 4b). The water passes through this spillway frontally through several rectangular internal breaches (Fig. 5). These characteristics block the upstream fish migration on this tributary. The engineering solution is the construction of a rectangular channel with a slope smaller than that of the river that is approximately 14\%. Thus, a rectangular channel of $1.5 \mathrm{~cm}$ thick concrete pallets (sheet piles) is fixed by the overflow threshold in front of the existing three breaches (Fig. 5). This concrete channel is also fixed to the concrete wall (Fig. 6) existing on the left bank of the river (tributary). 


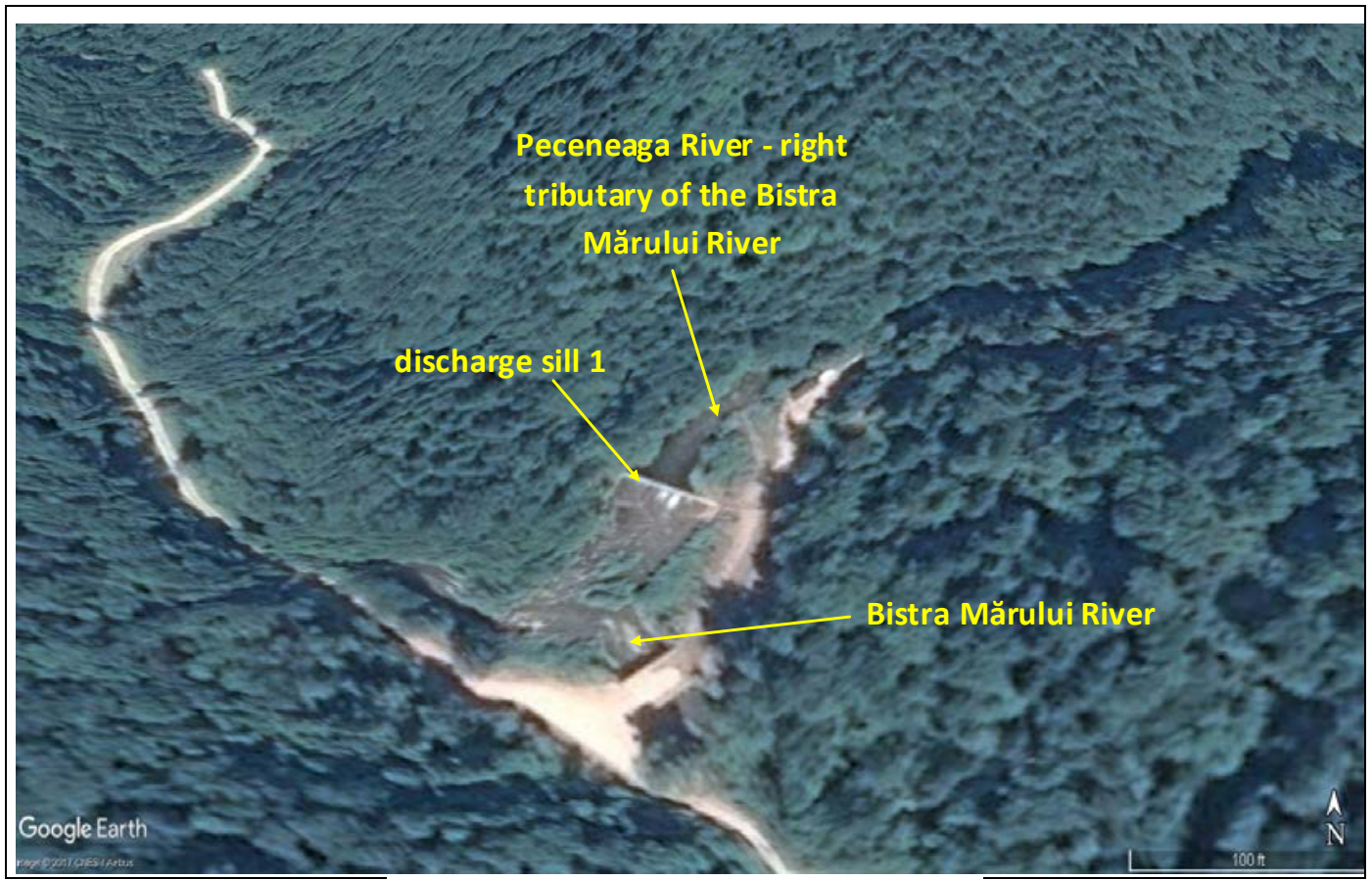

Figure 4a: Positioning the discharge sill at the confluence with the Bistra Mărului River.

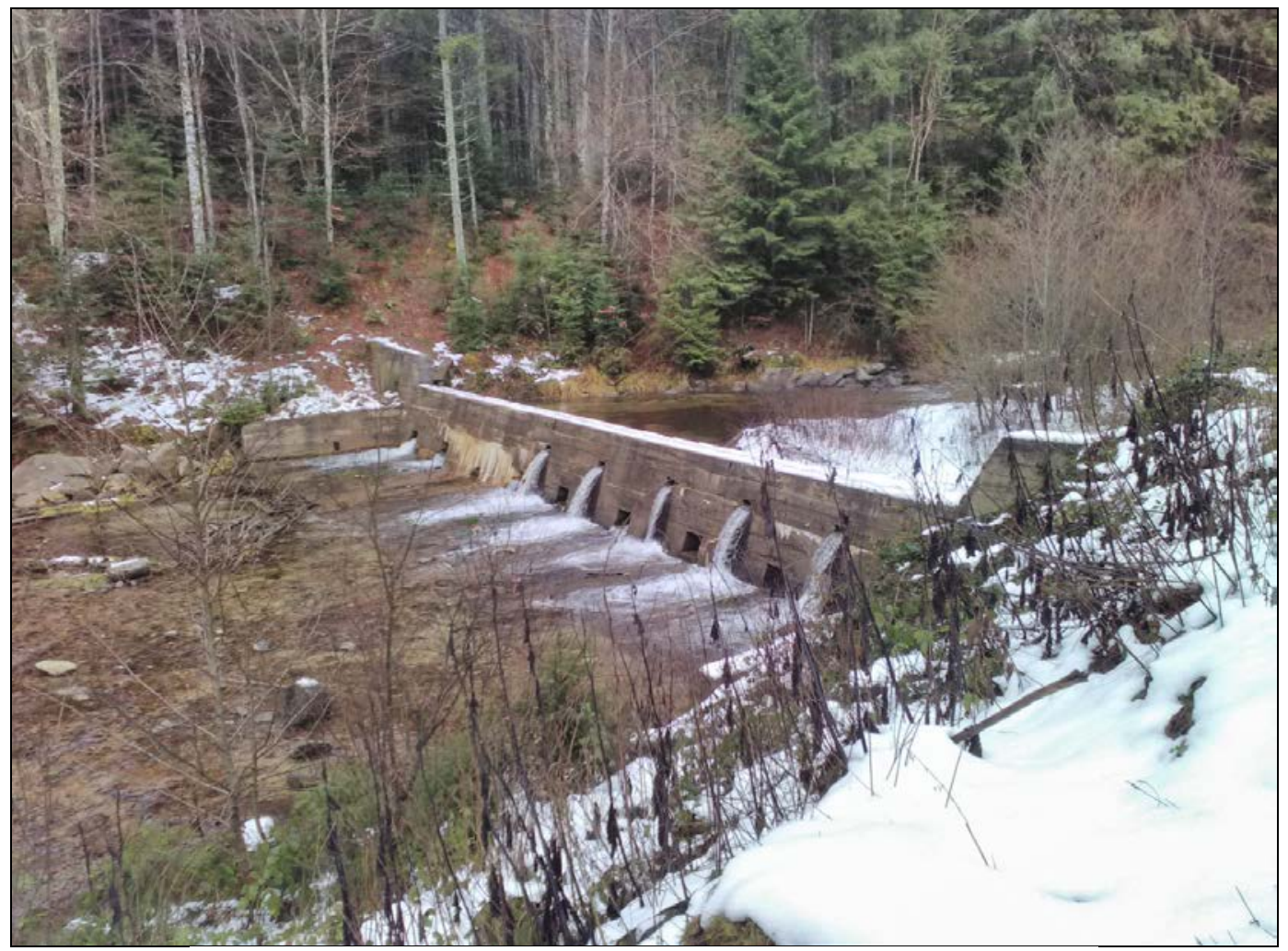

Figure 4b: The discharge sill on the tributary of the Bistra River. 


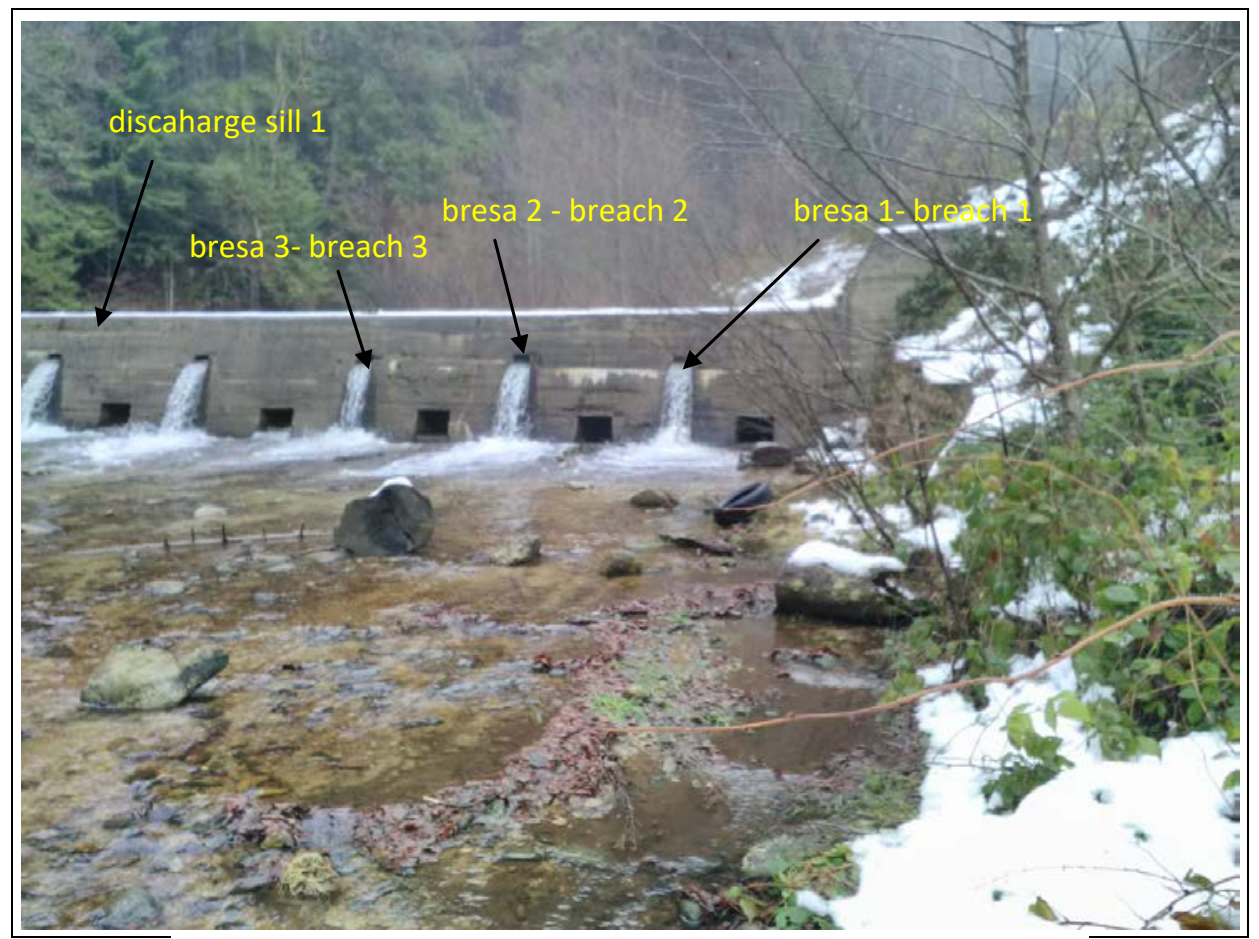

Figure 5: The three breaches located in the spill threshold 1.

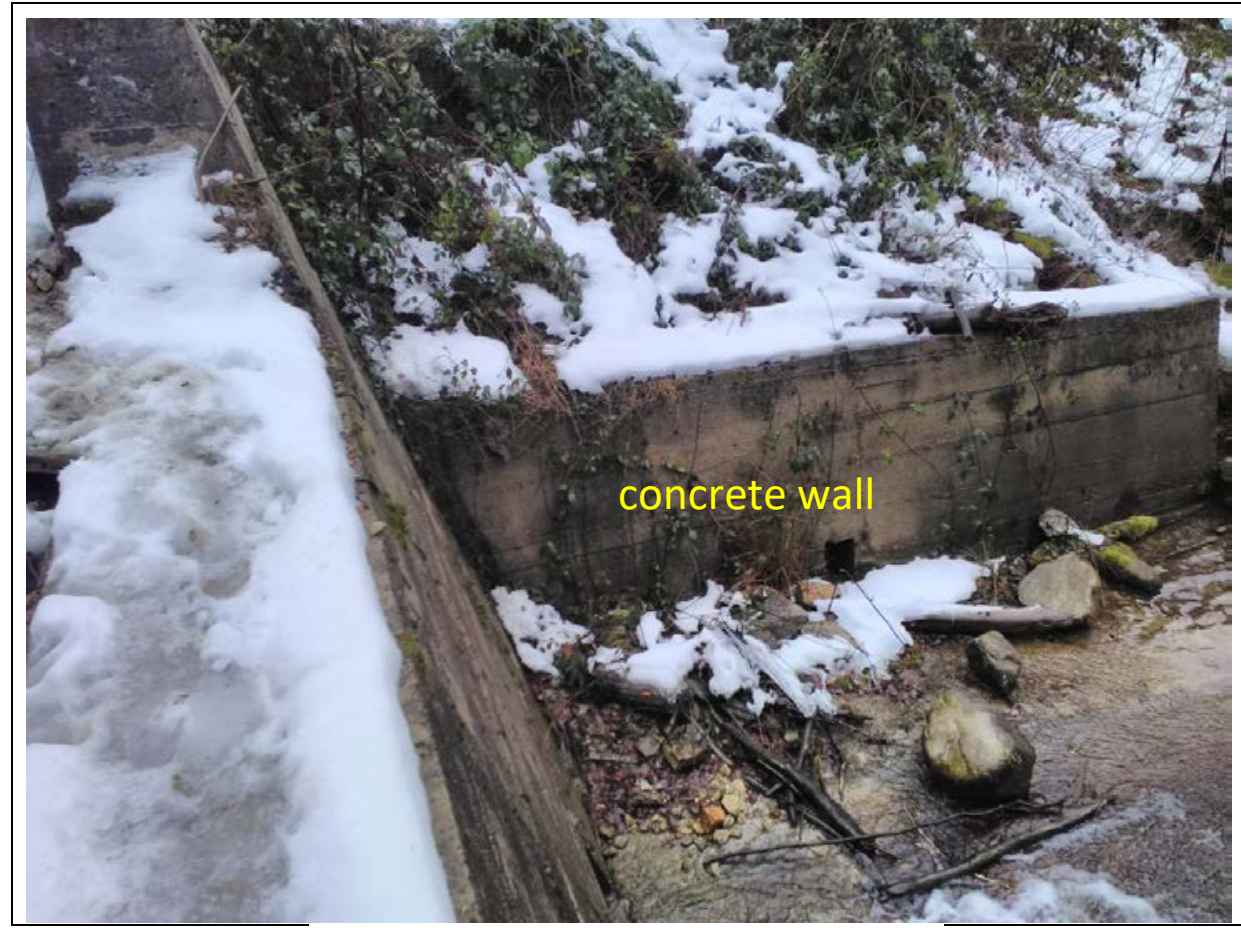

Figure 6: Positioning of the concrete wall at the left bank of the river. 
The concrete channel has a width of $40 \mathrm{~cm}$ and a hight of $30 \mathrm{~cm}$ and is fixed in metal dowels above the three existing breaches (1, 2 and 3). We mention that each bust is $25 \mathrm{~cm}$ high and $30 \mathrm{~cm}$ wide. In the sheet pile which is fixed directly to the spill threshold there are rectangular spaces of the same size as the existing breaches in the spill threshold (Fig. 7). The water level captured from the bridges 2 and 3 must overcome by a few centimetres the first breach (1) or at least the water level in the rectangular channel for fish migration occupy twothirds of the breach 1 (Fig. 7). Under these conditions, fish can easily migrate through the first breach. In the upper part of the first breech a rectangular parallelepiped must be constructed to light the brace 1. This rectangular parallelepiped is closed at the top (the crown of the spill threshold) and on the lower part (direct connection with the bust 1) with two rectangular glass surfaces transparent and very resistant (Fig. 8).

Before the fish migration canal goes over the concrete wall, an ihtyofauna rest pool is being built. This basin is actually a pocket of the fish migration channel and supports a concrete pillar (Fig. 7). The water inside the fish migration channel produces a remittal phenomenon within the breach 1 , reducing the existing speed in this breach, thus favouring the climbing of the fish through the breach and reaching them upstream of the spillway 1 . By this system, the breaches do not clog or obstruct they operate at normal capacity. Between the bust 1 and the bust 2 a metal fence is fixed to redirect the fish to the breach 1 (Fig. 7).

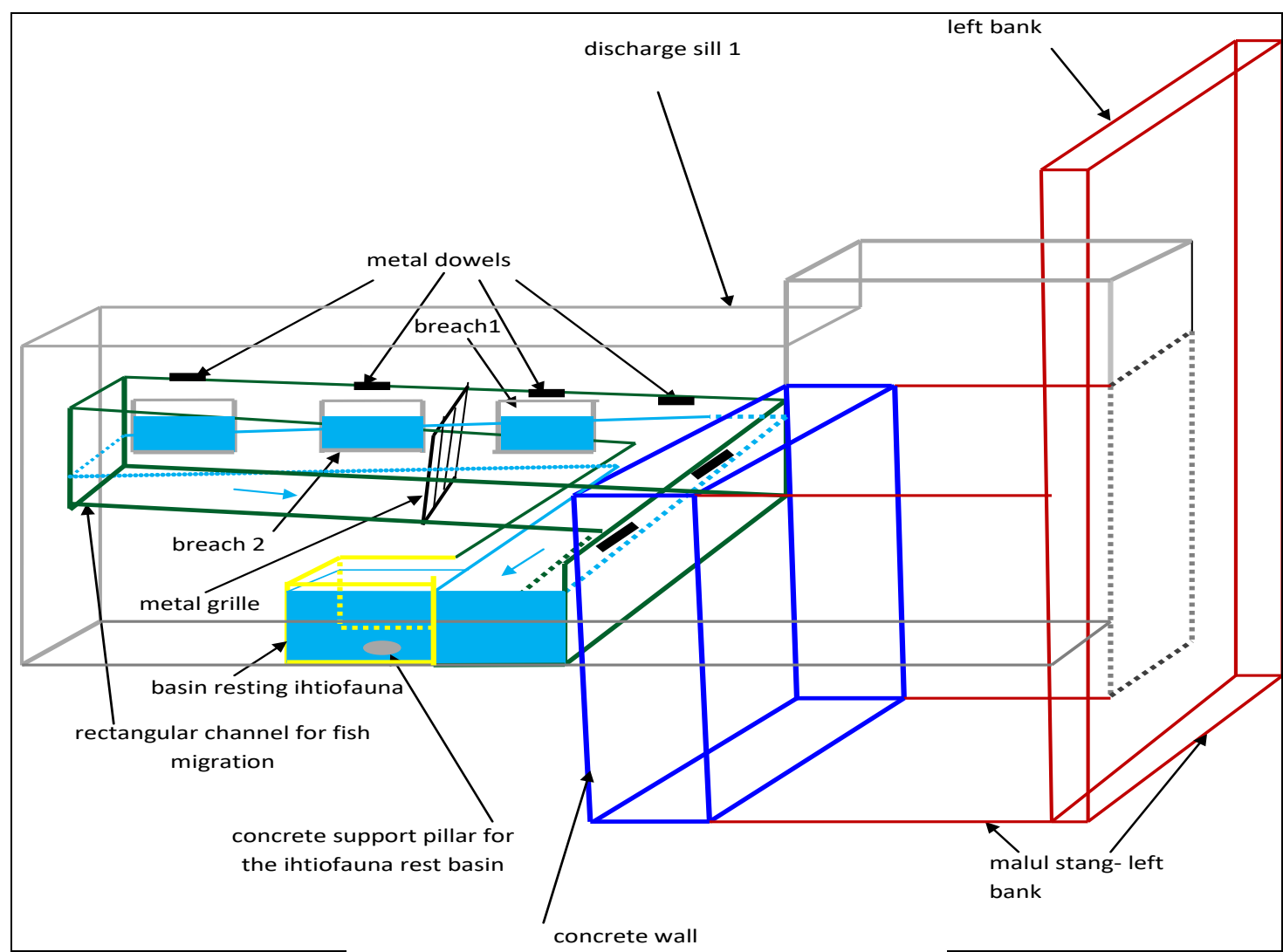

Figure 7: Positioning of the concrete channel on the spillway and the concrete wall - indicative scheme. 


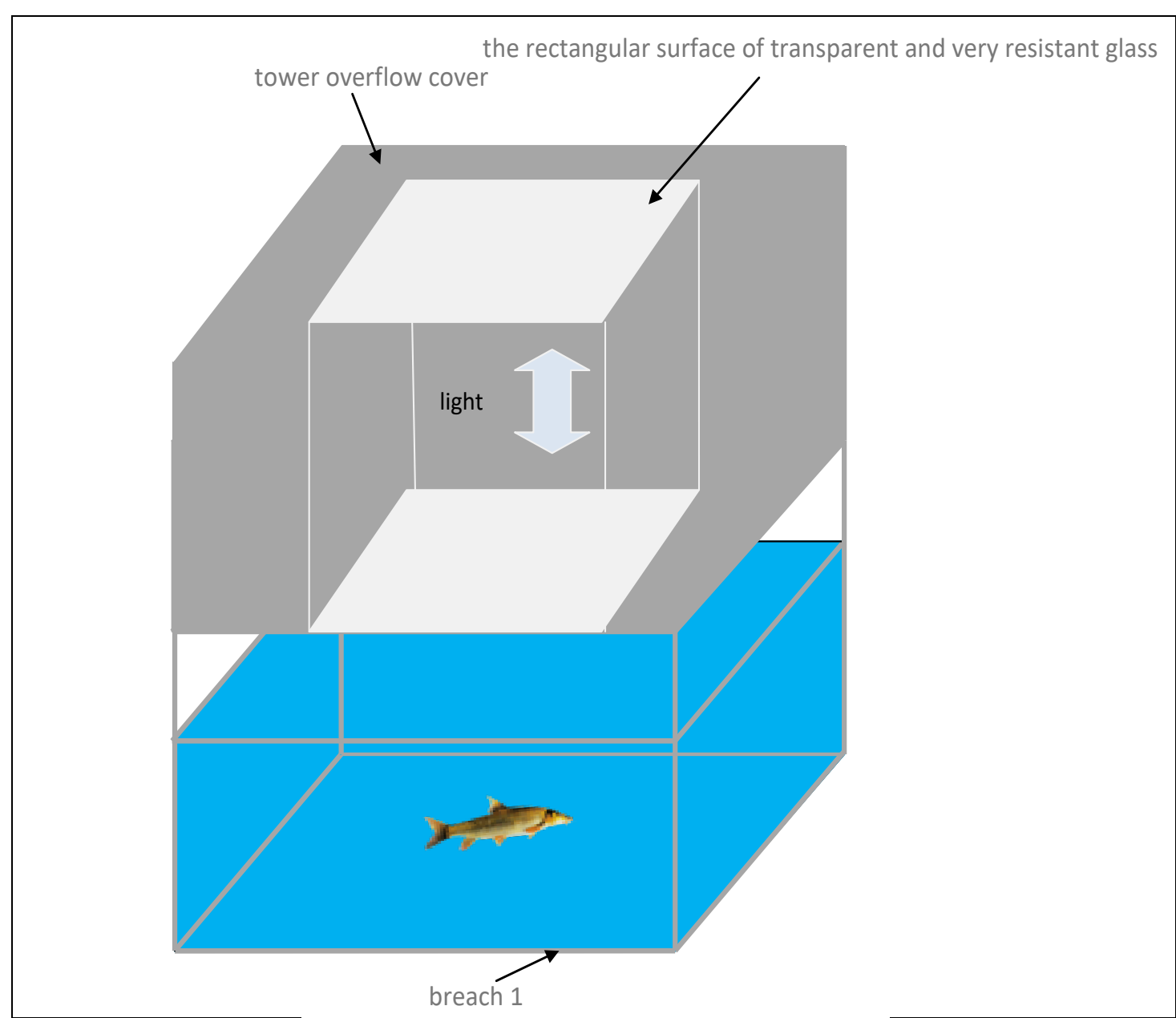

Figure 8: Positioning of the rectangular window

with a transparent and very resistant glass - indicative scheme.

After the entire concrete wall passes, the channel rises to the right until it reaches the water course (Fig. 9). The entire fish migration channel will have upper grids protecting the fish against poaching. The downstream end of the canal is in a reception pool where the water has a depth of about $30 \mathrm{~cm}$. In direct connection with the fish migration channel, fences are fed into the riverbed for redirecting the ichthyofauna to the receiving basin, thus allowing the fish to climb over the spillway 1. 


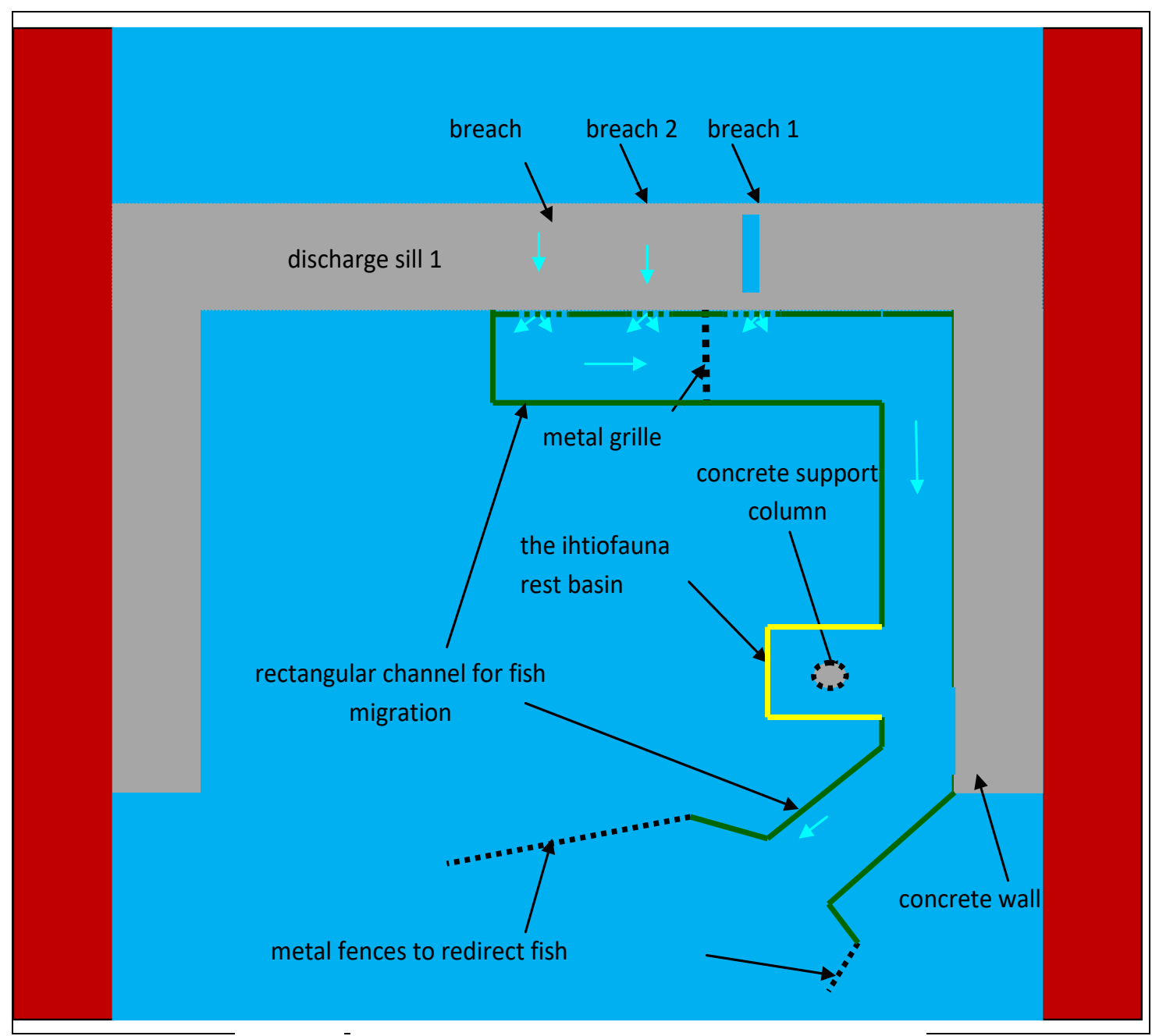

Figure 9: The overall scheme of the fish migration system over the spillway 1 - indicative scheme.

The fish migration channel could be assembled as modules and can be easily disassembled and assembled at another spill threshold if required. In case of exceptional floods, any damaged module can easily be replaced. The construction cost is not high and the realization of this fish migration system does not require very advanced technology that is actually useful for many countries. 
1.2 Spillways first category of proposed fish migration system solution - spillway number five study case downstream to tributary Peceneaga.

The length of the spill threshold (Fig. 10) on the Bistra River is about $10 \mathrm{~m}$ and the water speed is close to $0.8 \mathrm{~m} / \mathrm{s}$. The height of the spill threshold is $1.4 \mathrm{~m}$ and the width of the canopy of one $\mathrm{m}$ and the slope of the river in the area is about $12 \%$. At the threshold of the spillway 5 near the left bank of the river Bistra, a meter from the concrete wall is a breach (Fig. 11). The breach has a height of one $\mathrm{m}$ and a width of $40 \mathrm{~cm}$.

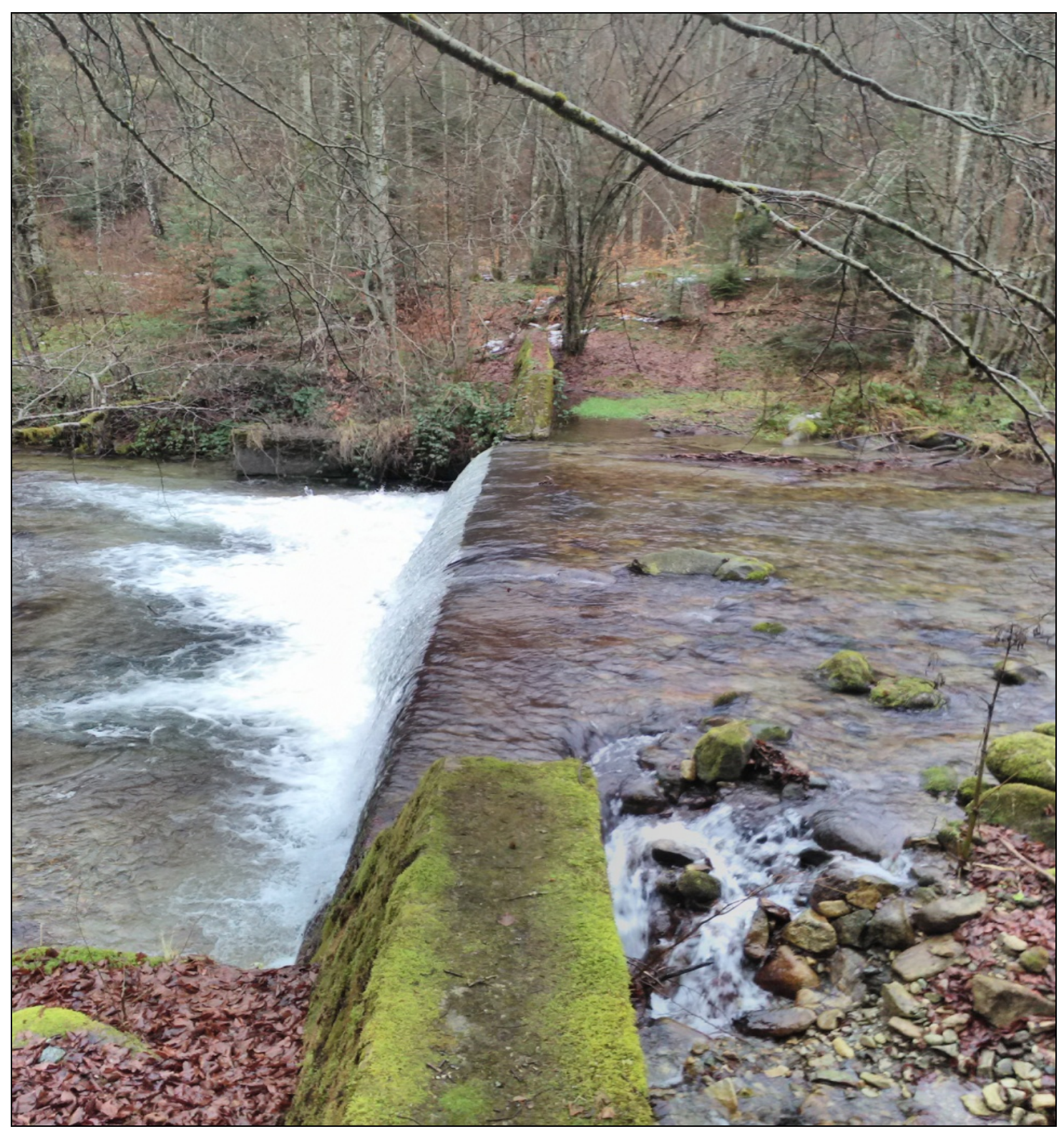

Figure 10: Discharge sill 5. 


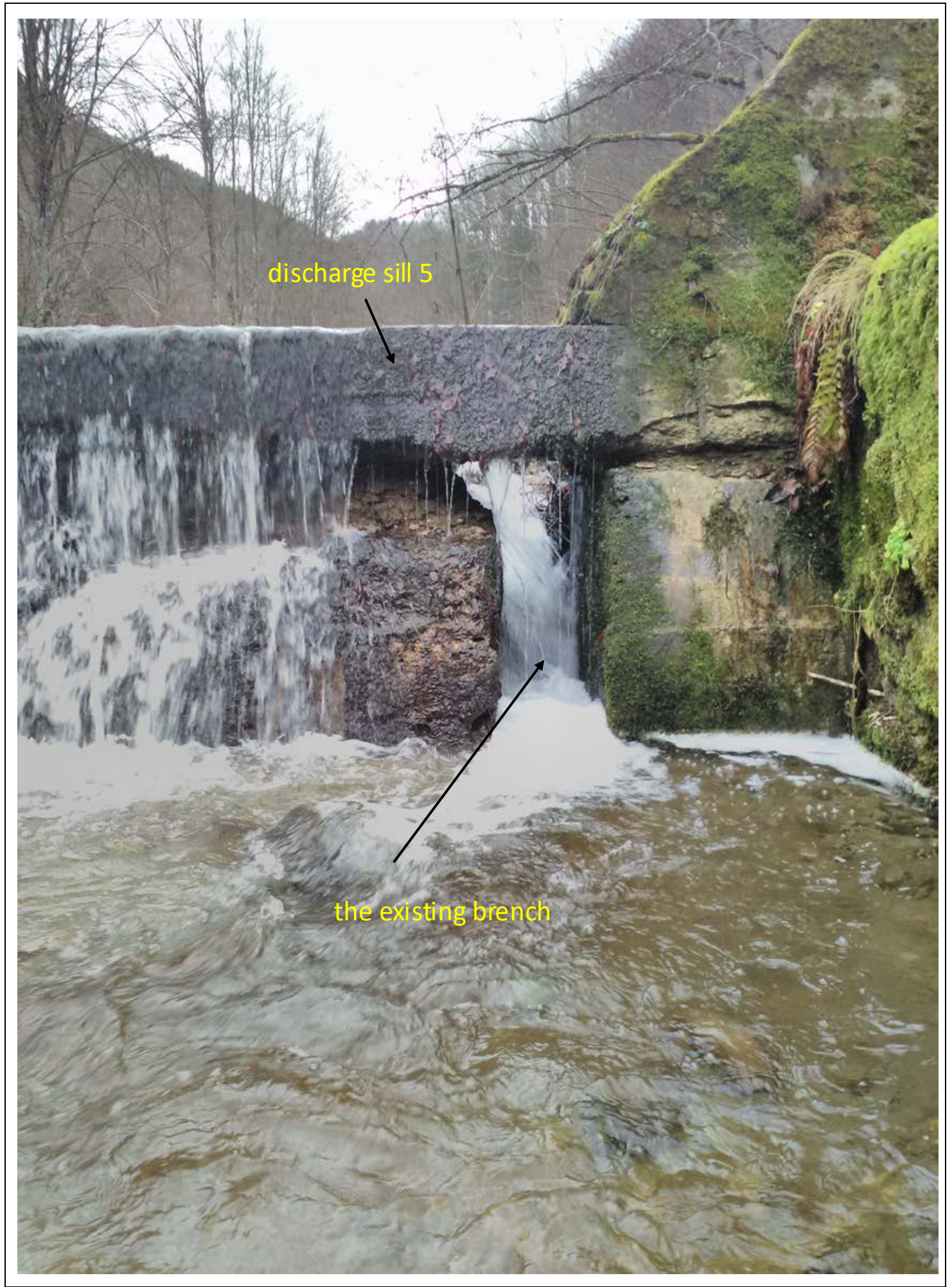

Figure 11: Positioning of the brench in the discharge sill 5. 
The water is captured in this breach by a natural semicircular slope with a large slope of about $60 \%$ (Fig. 12).

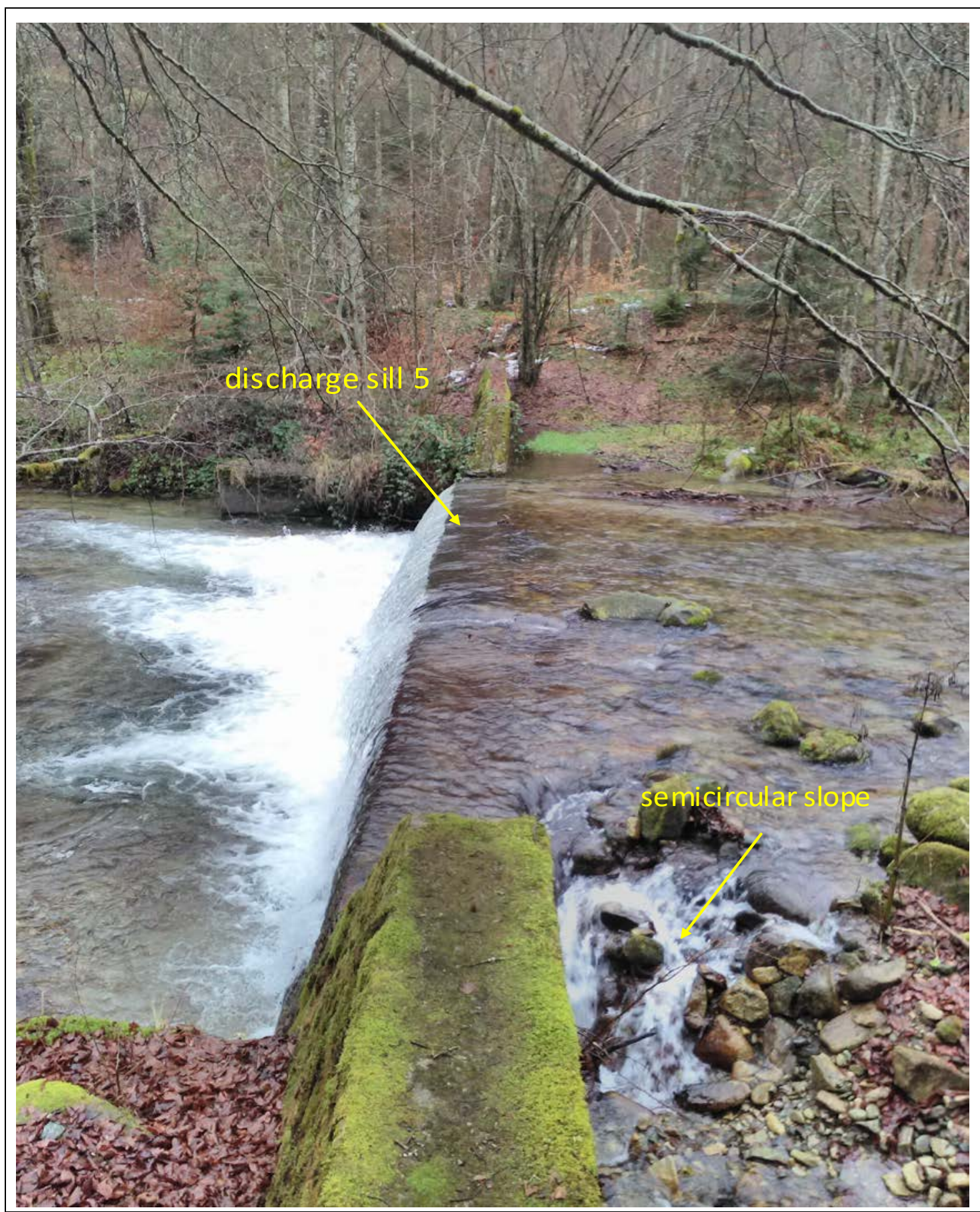

Figure 12: Capturing water in the existing breach with the help of the semicircular slope. 
A fish migration system must be developed within the existing breach. To reduce the slope, half of the slab must be filled with concrete. Fill it with a rectangular prism with a tilted surface (Figs. 13a and b).

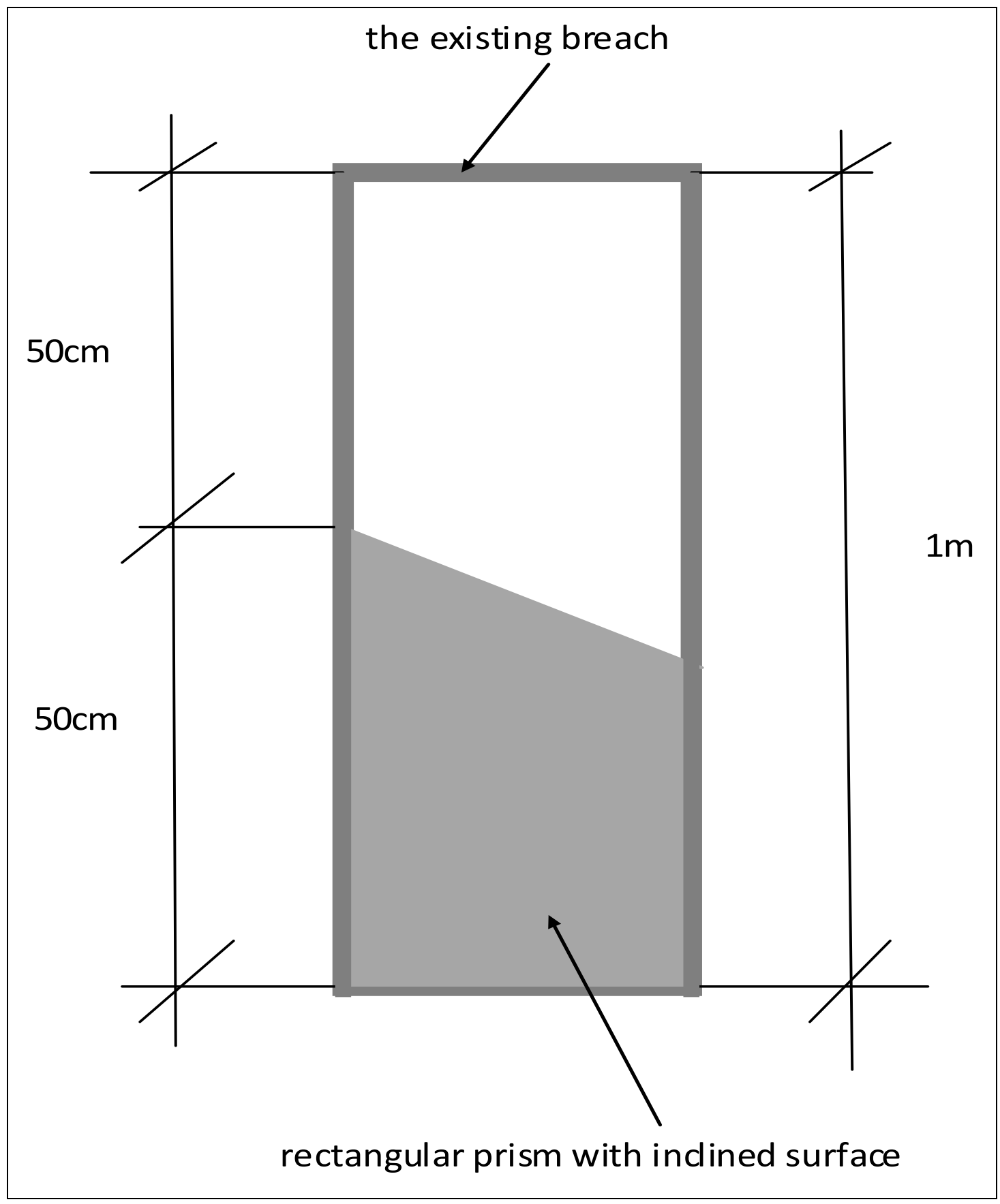

Figure 13a: Rectangular prism positioning with inclined surface

- cross section - indicative scheme. 


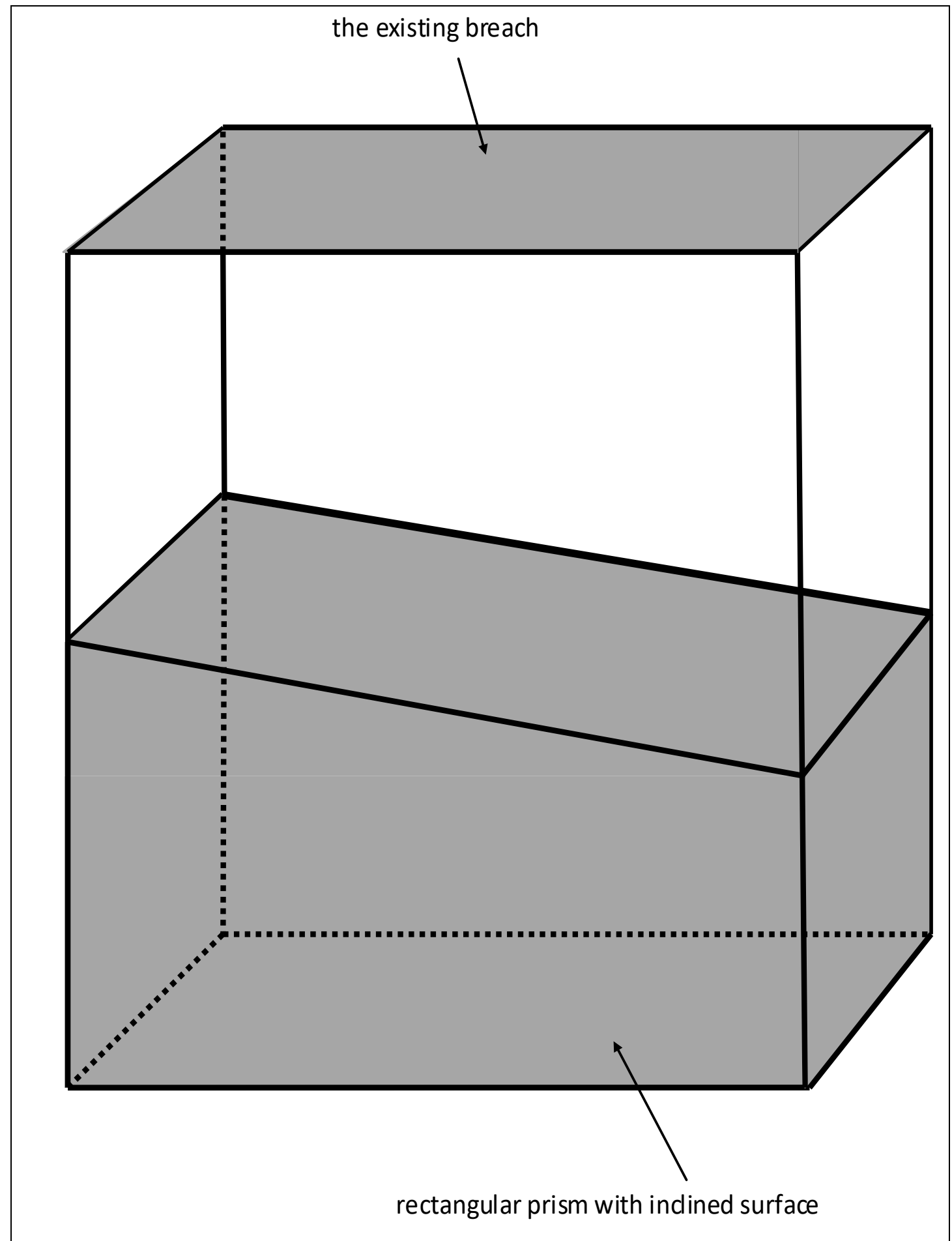

Figure 13b: Positioning the rectangular prism with a tilted surface - indicative scheme. 
The inclined surface will extend upstream of the discharge sill 5 to the upper level of the Bistra riverbed (Fig. 14). The river's riverbed must be arranged in a tilted plane on which will be fixed stones (Fig. 14). On the inclined surface, a concrete platform downstream of the discharge sill 5 will be fixed to the level of the Bistra riverbed (Fig. 14) and the downstream end will be fixed by a concrete pillar. The concrete platform will be fastened by a concrete pillar and at the upstream end (Fig. 14).

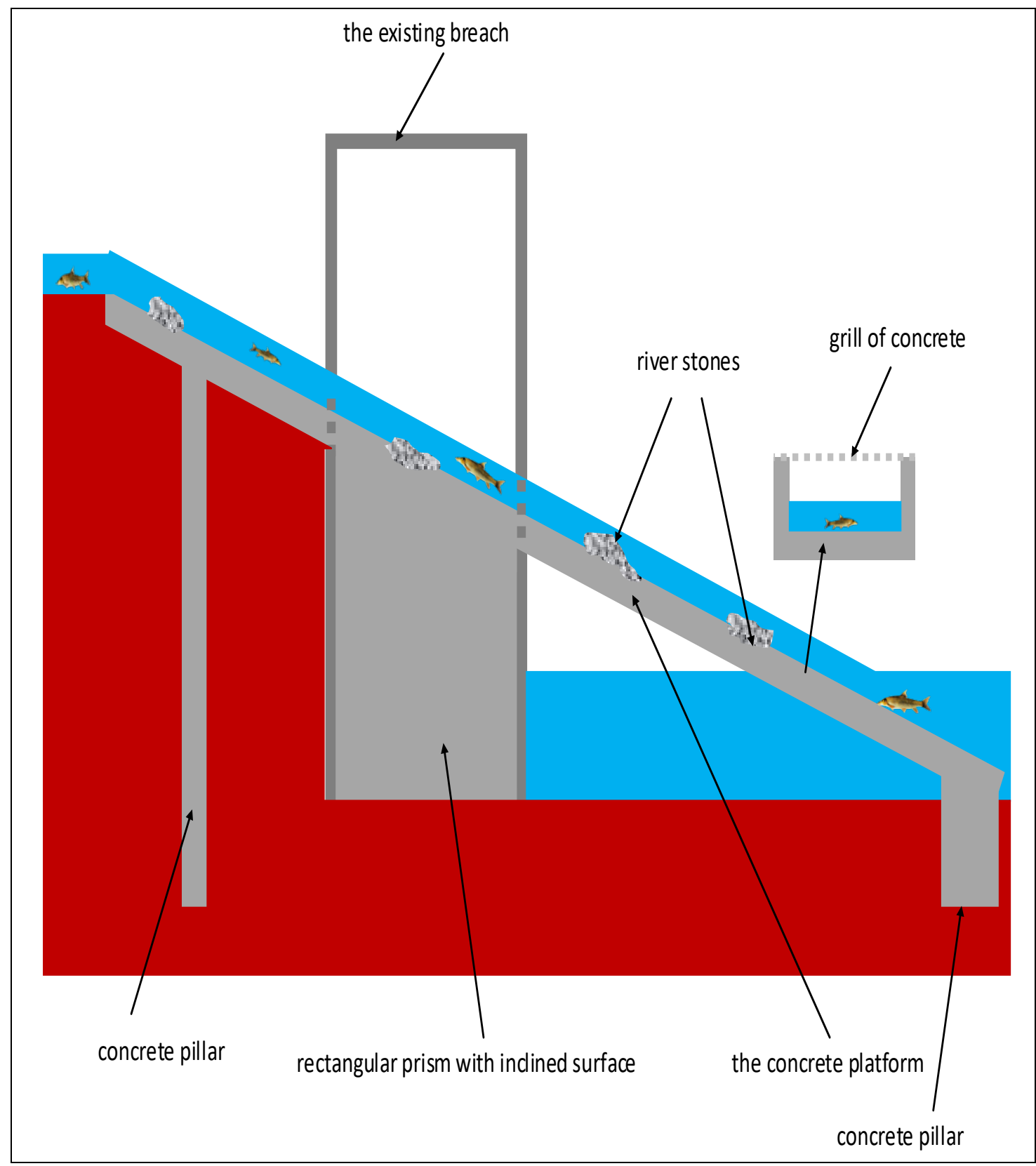

Figure 14: Positioning of the concrete platform upstream and downstream of the discharge sill 5 - indicative scheme. 
Downstream of the discharge sill the concrete platform will be framed by concrete parapets and above it will have a concrete grid as a defence against the poachers (Fig. 14). Upstream, the slopes limiting the concrete platform that the fish pass upstream - downstream of the spillway are stabilized with a concrete layer on which river stones are attached (Fig. 15). The slopes cannot be crossed by the fish.

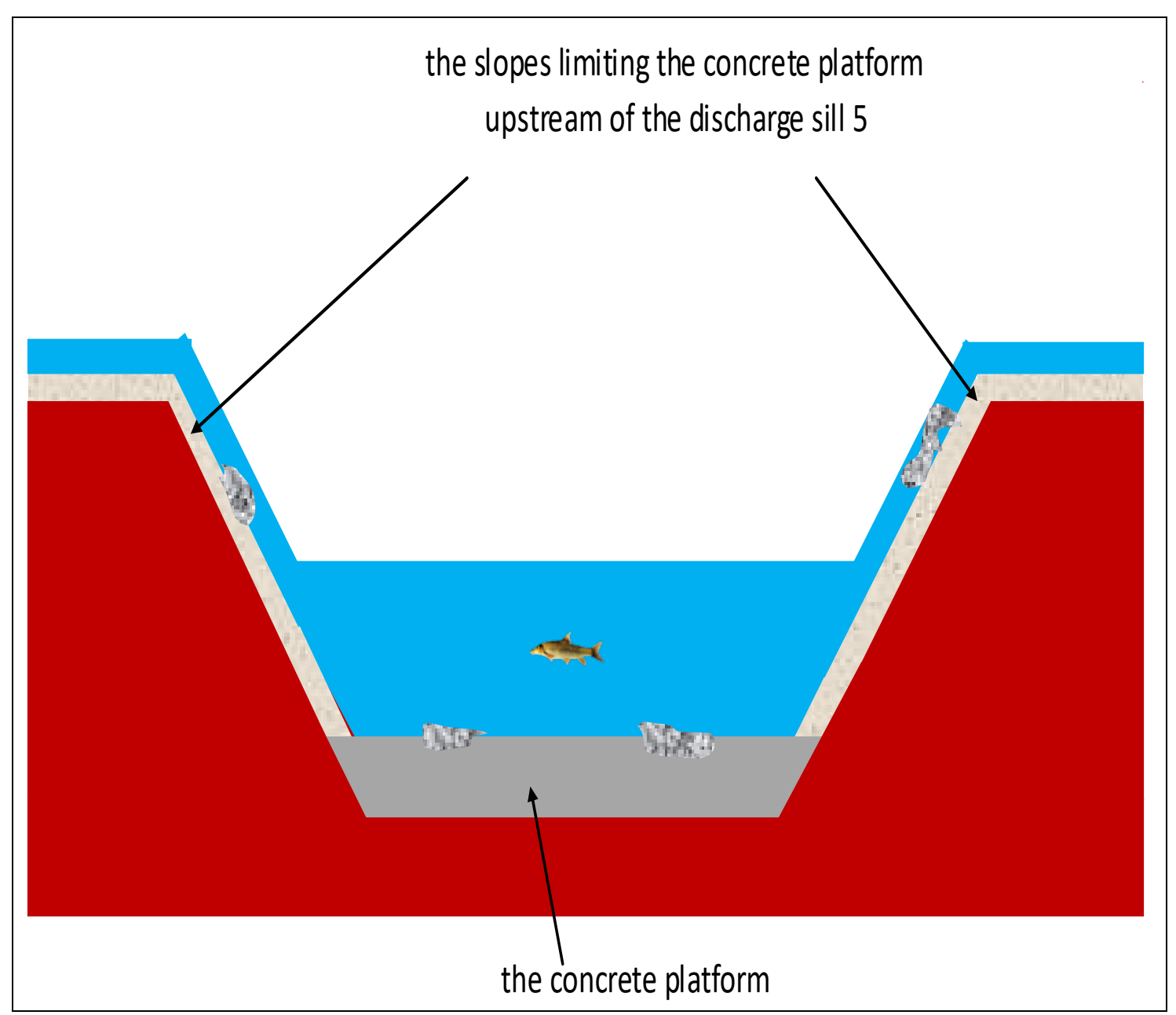

Figure 15: Reconstruction of the concrete platform slopes upstream of the discharge sill 5 - indicative scheme

This solution category provides a safe migration solution to the upstream fish, does not affect the structure and functionality of the spillway 5 and resists flooding due to the structure of the fish migration system. 
2. The middle Bistra Mărului River, fish migration solution across the Poiana Mărului Dam.

The dam of Poiana Mărului Lake (Figs. 16a, b, c) was conceived from rockfill with clay core. The emptying of the reservoir is carried out through a pressure inlet manifold up to the house of the lake emptying valves and is equipped with two flat valves in the housing and a free-standing gallery after the house of the valves. The discharge rate is about $159 \mathrm{~m}^{3} / \mathrm{s}$. A bypass duct leaves the house of the valves and provides a service flow of one $\mathrm{m}^{3} / \mathrm{s}$ which in turn feeds MHC from the base of the dam. (http://www.hidroconstructia.com/dyn/2pub/proiecte_det.php?id=38\&pg=1)

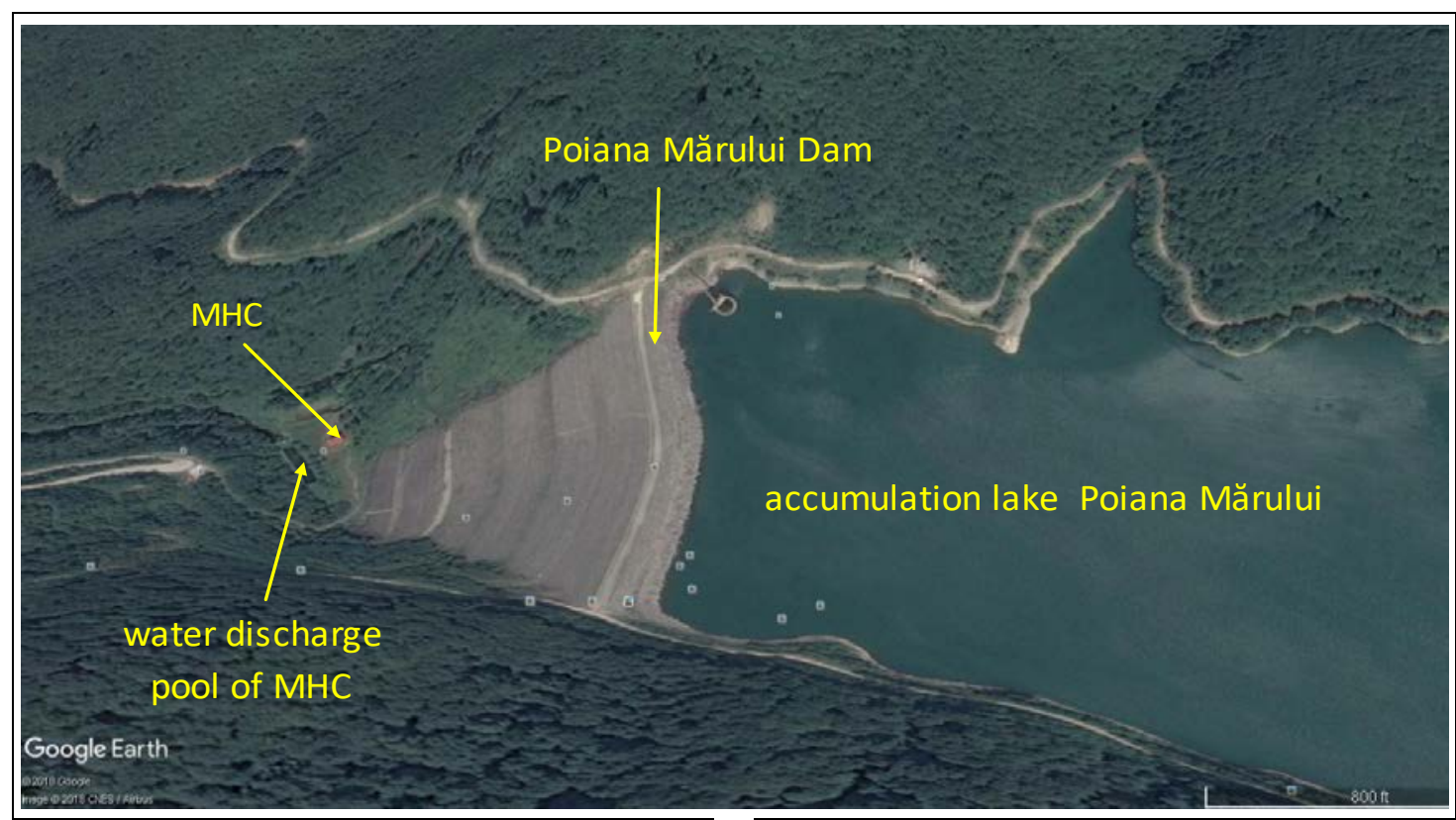

a).

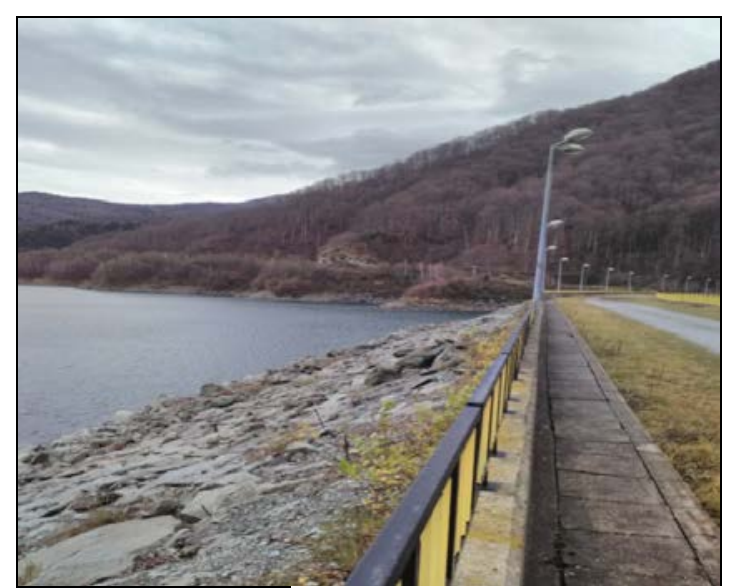

b) upstream.

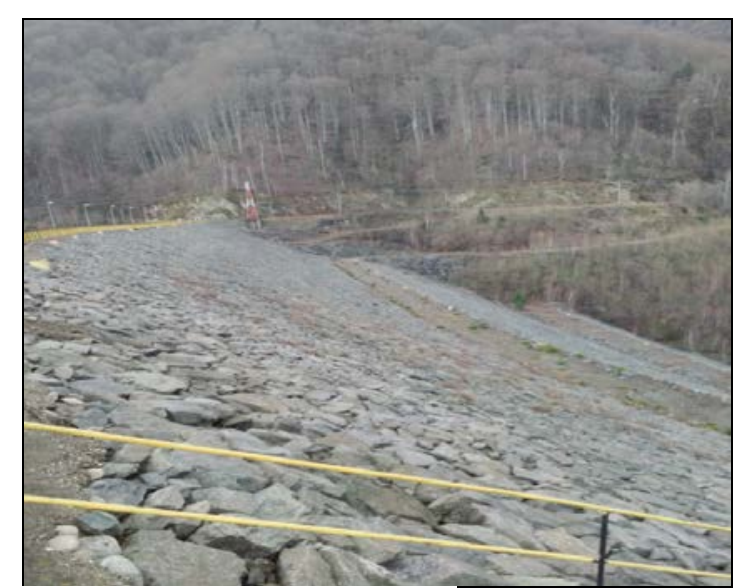

c) downstream.

Figure 16a, b and c: Poiana Mărului Dam. 
After feeding the MHC the water is discharged into a rectangular parallelepiped basin (Fig. 17) and from this basin the water returns to the river bed of Bistra Marrului (Fig. 17).

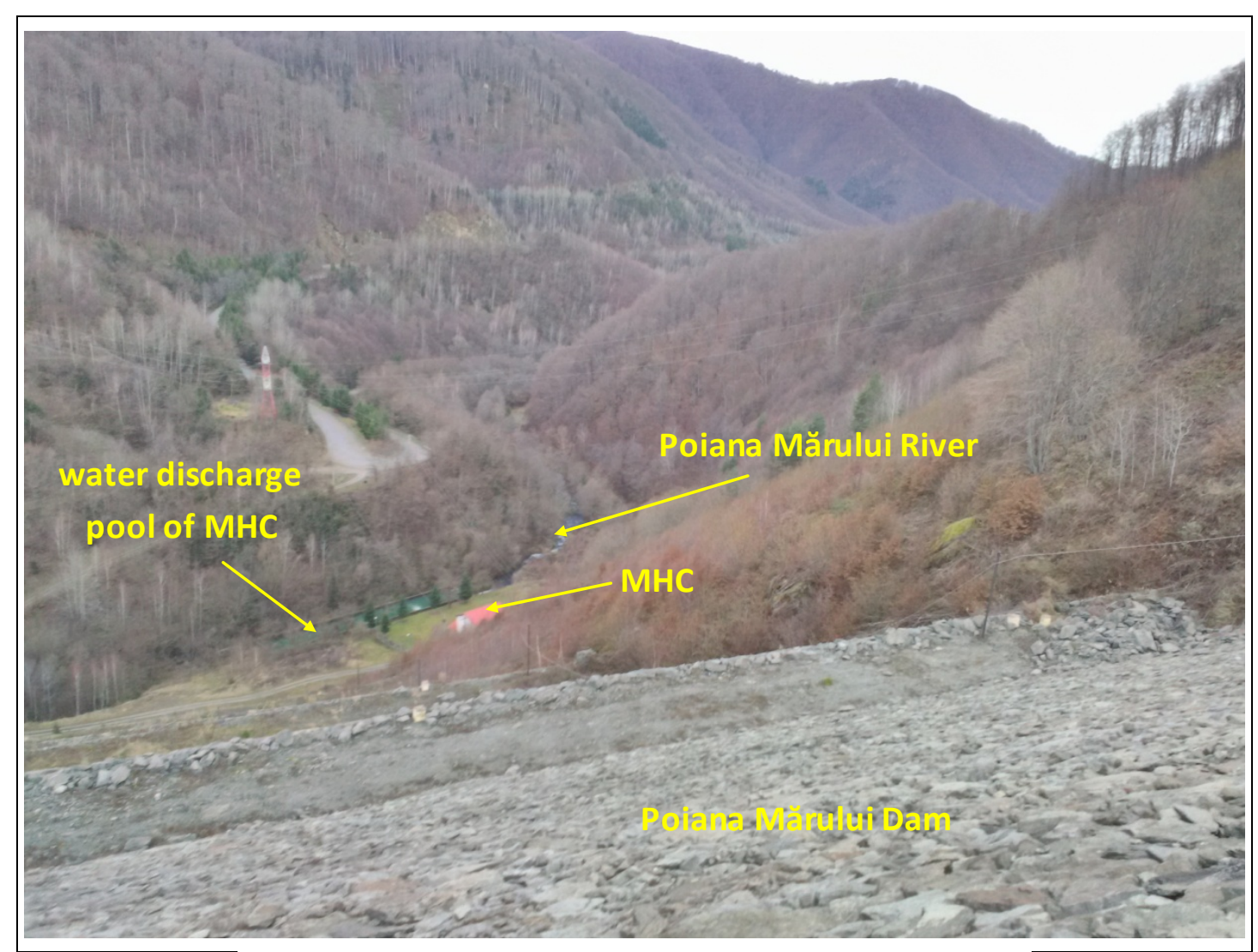

Figure 17: Positioning of the water discharge basin in MHC.

Downstream of the discharge basin (about five m), the river will be divided into half with a five $\mathrm{cm}$ thick concrete sheet pile and eight $\mathrm{m}$ long. In this basin must be a break in which a metal pipe with a manual or automatic valve must be fitted. On the left bank there will be a concrete sheet pile and a new concrete sheet pile will be attached on the first concrete sheet pile. The second concrete sheet pile will divide the river in half (Fig. 18). In the left side of the river divided by the concrete sheet pile, a rectangular parallelepiped basin of concrete (basin 1, quenching) will be built where the water discharged out of the pipe. The flow rate of the pipeline is about $0.5 \mathrm{~m}^{3} / \mathrm{s}$. River flow in the area is about one $\mathrm{m}^{3} / \mathrm{s}$. From the first basin, a hydraulic jump (40 cm) will connect a second basin (basin 2) (Fig. 18). The basin 2 will be parallelepiped and will be below the river level (Fig. 19). 


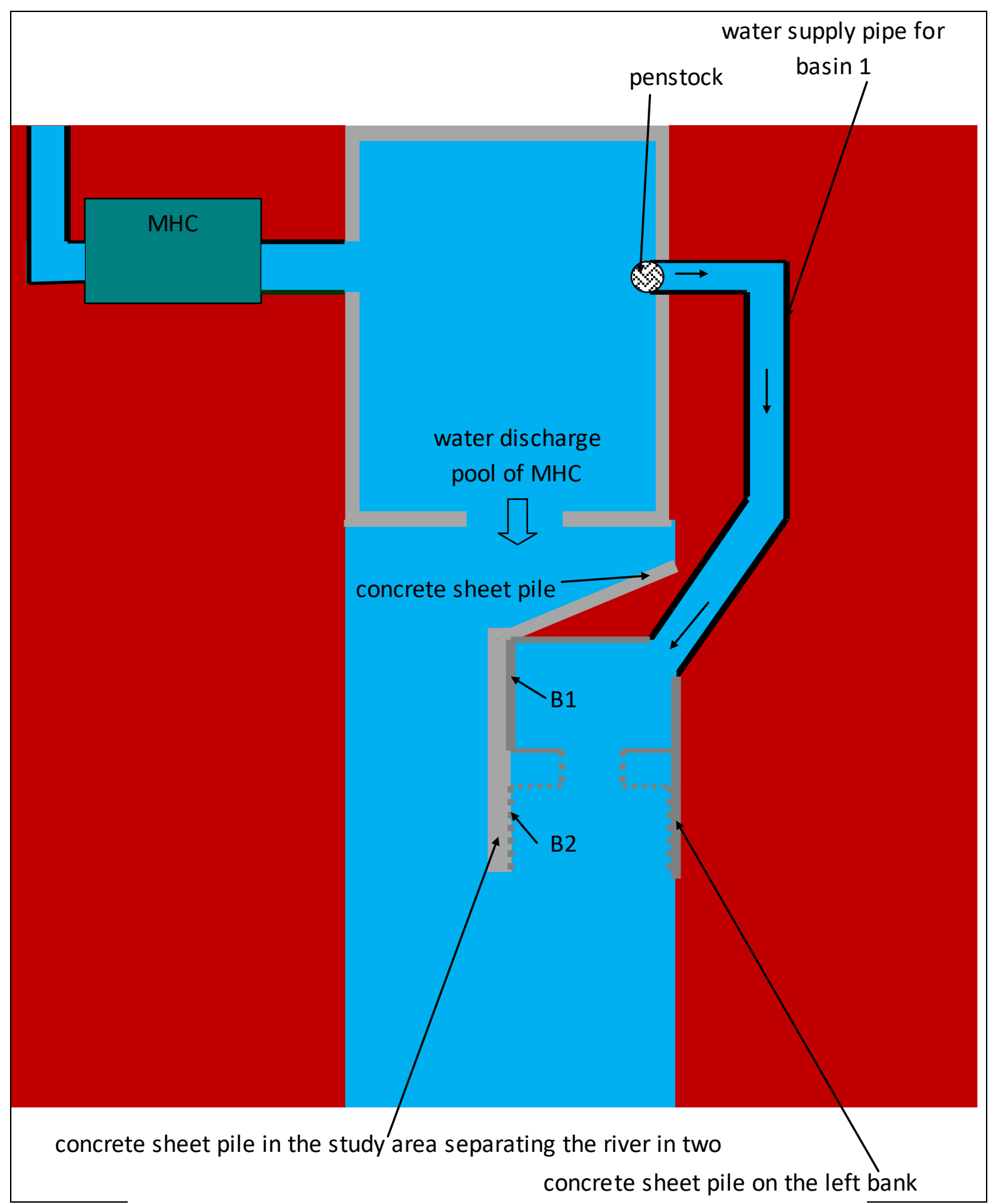

Figure 18: Positioning of the metallic pipe feeding the basins B1 and B2 and positioning of the two basins B1 and B2 - indicative scheme. 


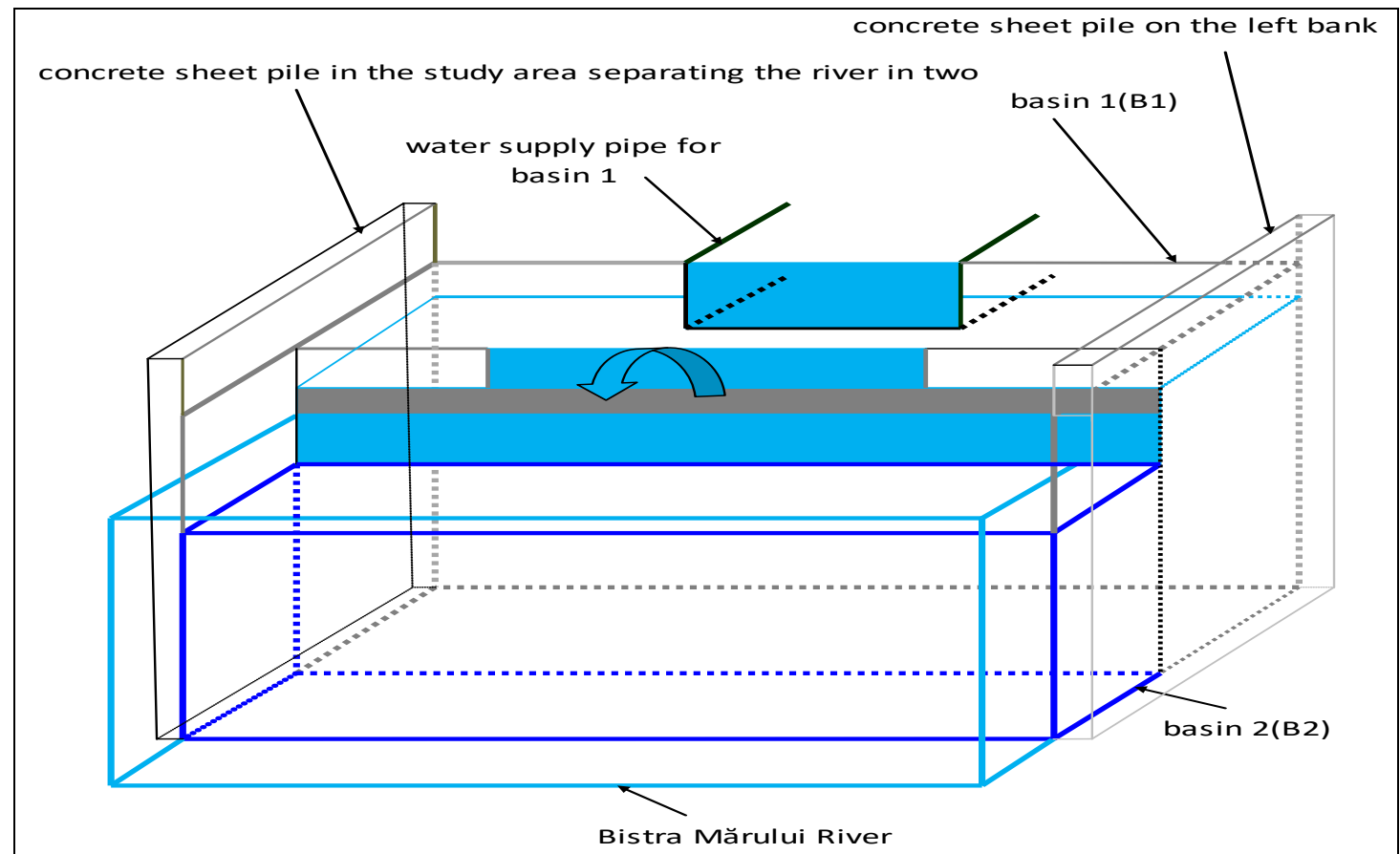

Figure 19: The link between basin 1 and basin 2 - indicative scheme.

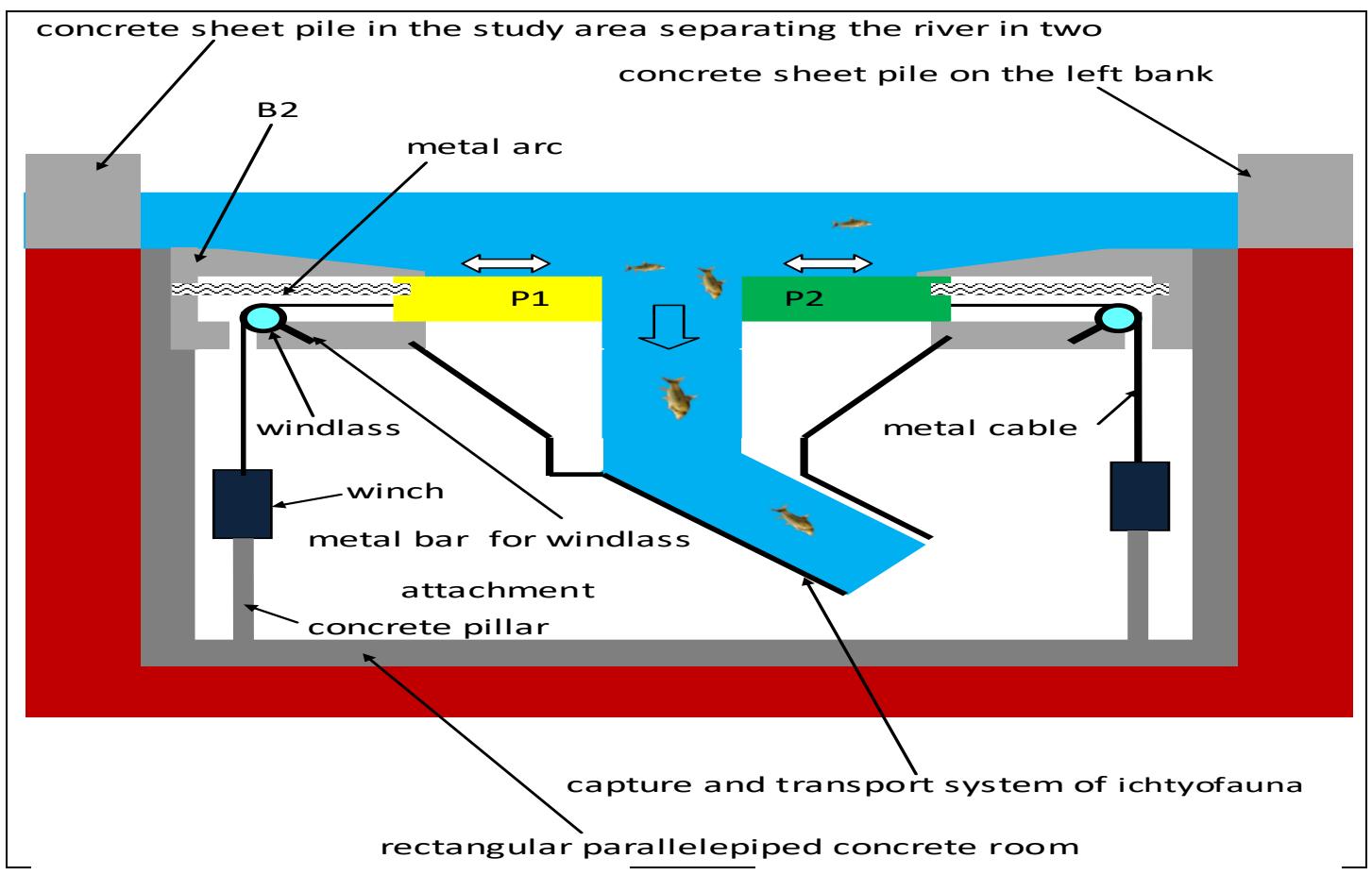

Figure 20: Placing of moving P1 and P2, windlass and trolleys inside the rectangular space - indicative scheme. 
Approximately $20 \mathrm{~cm}$ of water is above the basin 2, in fact the Bistra Mărului River level is in the study area. The base of the basin 2 should be approximately $30 \mathrm{~cm}$ thick and include two moving concrete slabs in a horizontal plane. The upper part of the basin 2 is shaped like a funnel for water capture. A parallelepiped rectangular special room must be constructed under the base of the basin 2. There is an electric winch next to the downstream end of the P2 concrete sheep pile. There is an electric winch next to the upstream end of the P1 (concrete sheep pile). Next to each electric winch is a metal bar on which a windlass is welded. Over the two windlass are placed the two wires of the electric winches that will be fastened by the two mobile plates P1 and P2 (Fig. 20).

The metallic springs are fixed to the concrete sheet piles P1 and P2 and to the walls of the underground rectangular space (Figs. 20 and 21). At the moment when the windlass is moved due to an ichthyofauna sensor, the concrete plates are moved with horizontal movement. During the plate movements, the springs contract and de-contract when the concrete sheet piles P1 and P2 return to the original position. The downstream end of the underwater capture and transport system of the ichthyofauna will be directly linked to a horizontal parallelepiped subterranean basin located horizontally. This does not have a vertical surface (Fig. 22). The downstream end of the underground capture and transport system of the ichthyofauna fixes on a metal arch a thin transparent and resistant plastic sheet pile that allows the water and the ichthyofauna to penetrate into the horizontal rectangular parallelepiped basin located in the horizontal plane (Fig. 22).

Inside the rectangular parallelepiped basin located horizontally, a parallelepiped of resistant rubber slides into the horizontal rectangular parallelepiped basin. Between the rubber parallelepiped and the top of the right (underground) basin located in the horizontal plane remain approximately $20 \mathrm{~cm}$. These $20 \mathrm{~cm}$ remain the full length of the rectangular parallelepiped basin in the inclined plane. From the upper surface of the rectangular parallelepiped basin located horizontally, a metal reinforced bar is fastened. From this bar through two metal rings, the movable parallelepiped of rubber is fixed. The rectangular parallelepiped basin located horizontally will be extended also in inclined plane (Fig. 22). A metal cable (Fig. 22) should be attached to the rubber parallelepiped. The right-angled (subterranean) parallelepiped basin located in the horizontal plane will exit the underground and be fixed on concrete pillars and extend in an inclined plane and then horizontally up to the dam (Fig. 23). The upstream end will be secured by a concrete pillar (Fig. 23). The movable rectangular parallelepiped of rubber will be towed up to the upstream end of the rectangular parallelepiped basin located horizontally by a winch located on a concrete pillar on the bank of the accumulation lake Poiana Mărului (Fig. 23). At the moment of closing the transparent and resistant plasterboard with a sensor, the winch is automatically placed on a concrete pillar on the bank of the accumulation lake Poiana Mărului. Detaching the water from the fish migration system will be done through a free-standing rectangular canal. 


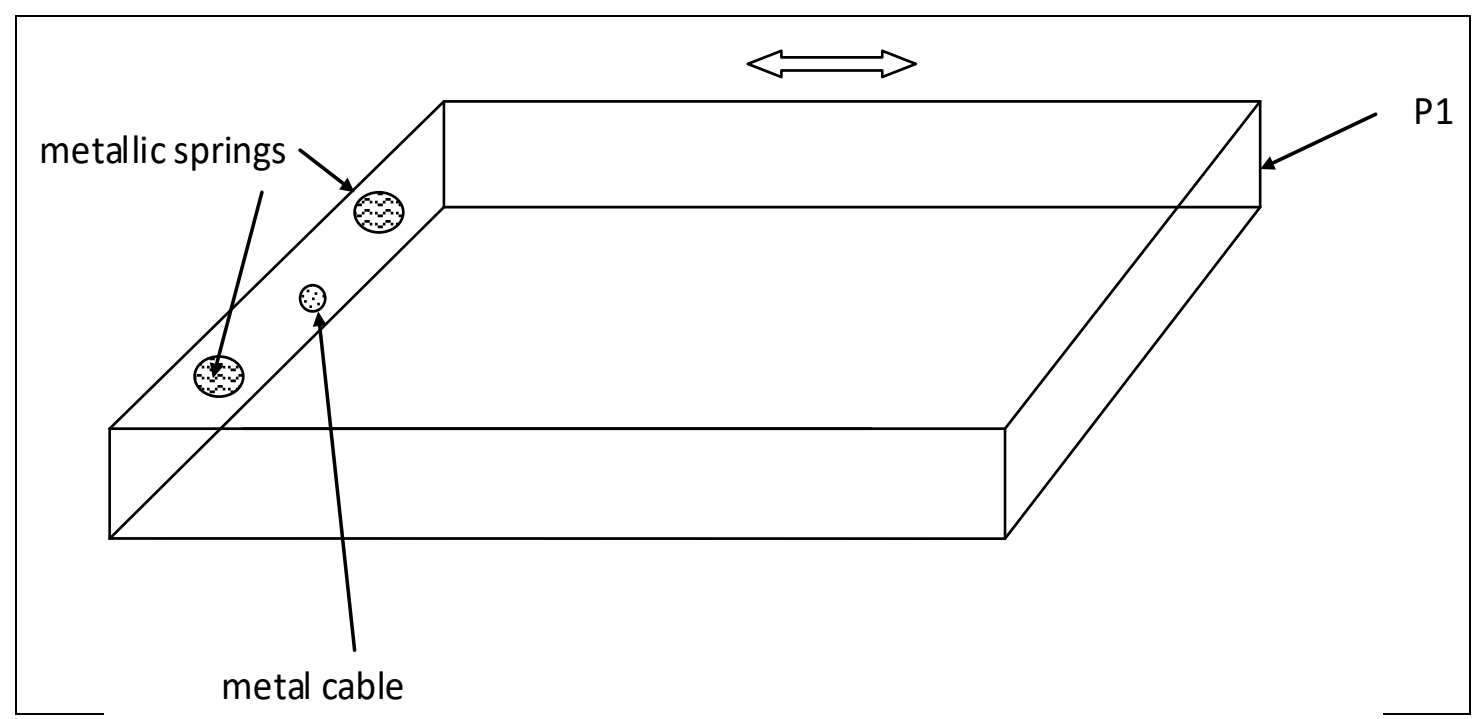

Figure 21: Positioning the metallic springs and the metal cable - indicative scheme.

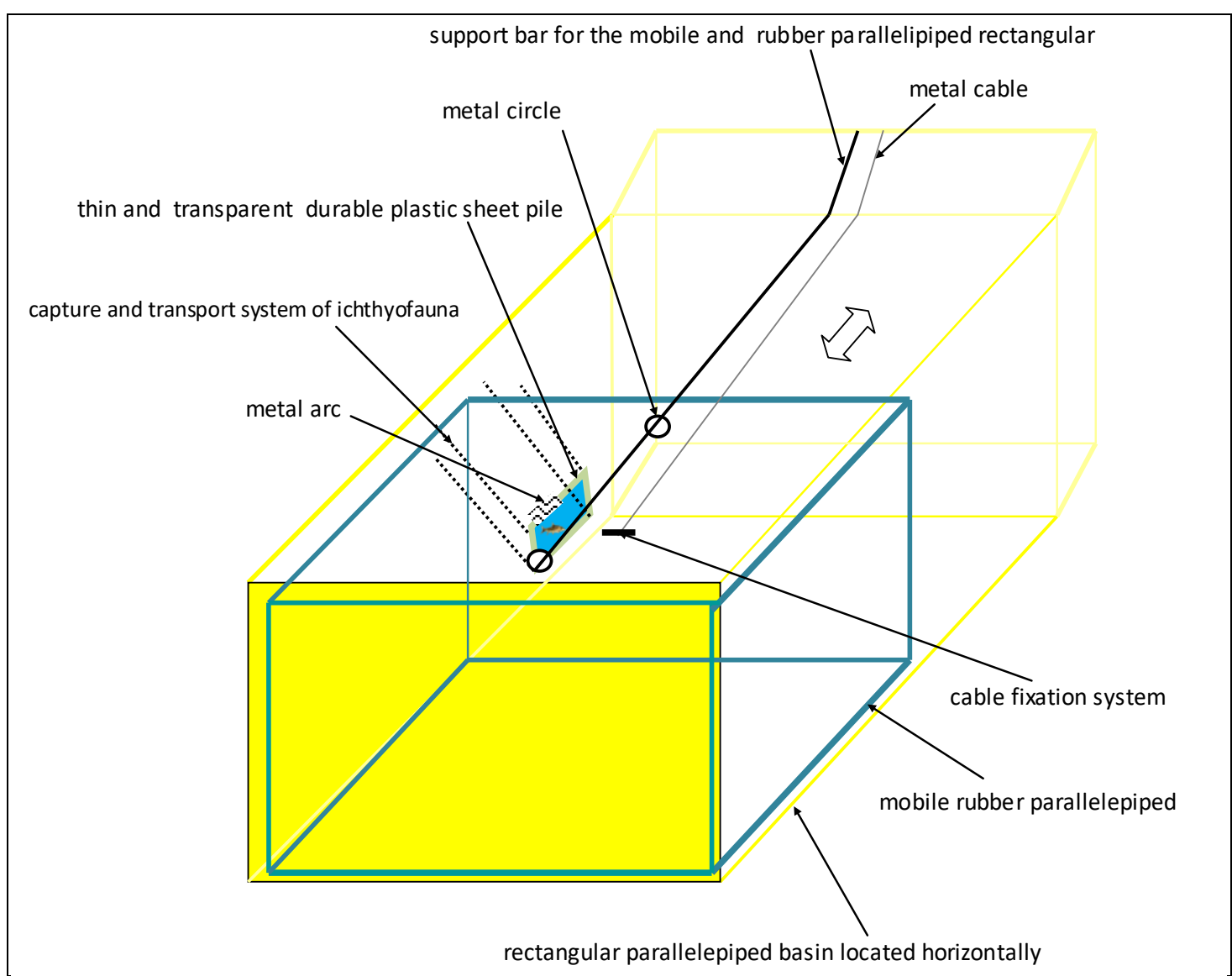

Figure 22: Positioning of the mobile rectangular parallelepiped from rubber-indicative scheme. 


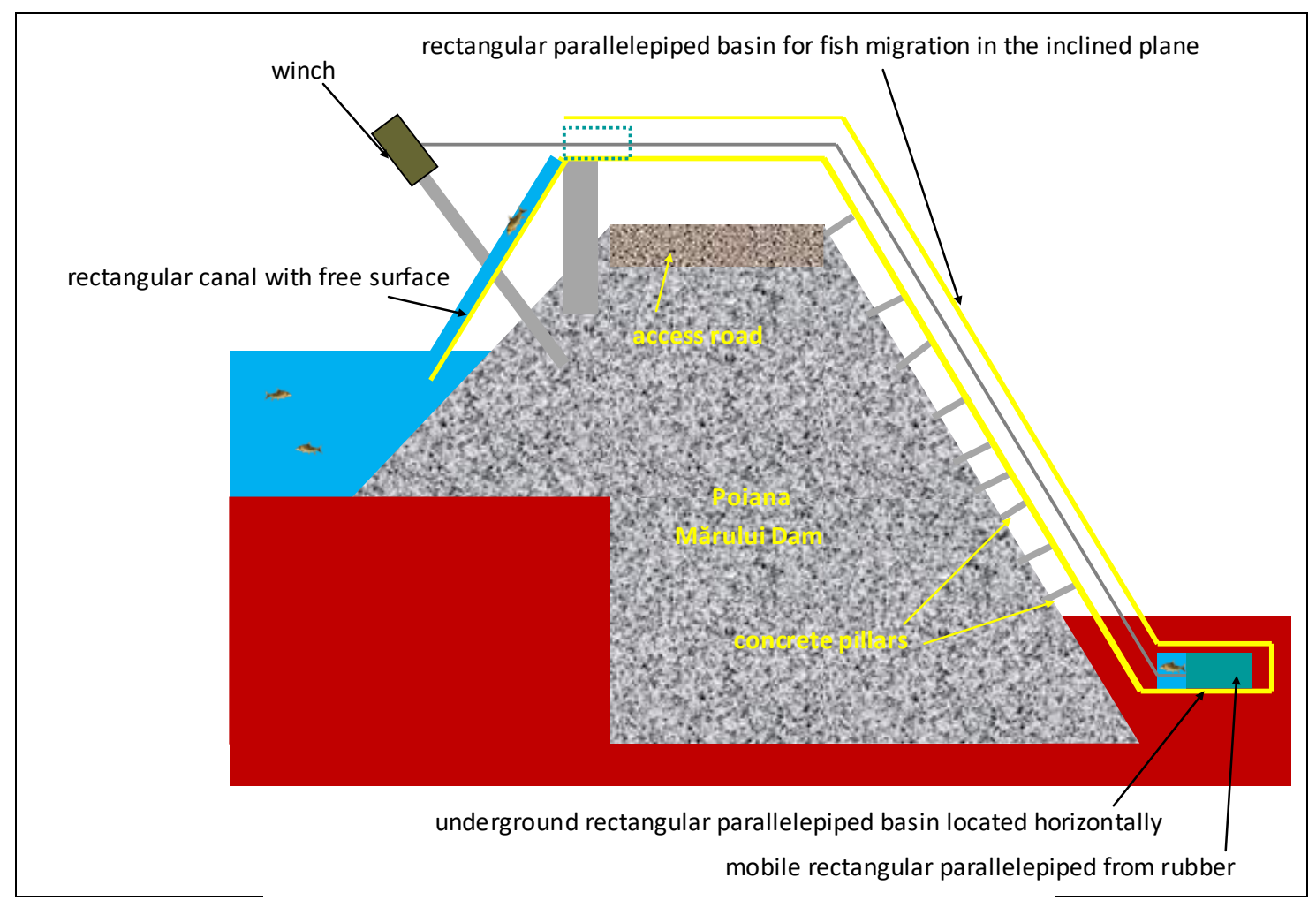

Figure 23: Positioning the rectangular parallelepiped basin on the Poiana Mărului Dam - indicative scheme.

From the back surface of the rubber parallelepiped that pushes the fish into the reservoir Poiana Mărului, a metal cable is attached which belongs to a winch inside the righthand parallelepiped located horizontally. After the caulking parallelepiped reaches the maximum level and pushes the fish into the reservoir, this winch pulls the mobile parallelepiped in the initial position after five seconds after running the entire length of the fish migration system.

The hydraulic jump between basin B1 and B2 draws the ichthyofauna before the water is discharged from MHC, which is an important fact. For redirecting fish, low amperage systems (generators) can also be fixed in the riverbed as the safety of reaching the fish above basin B2 is maximum. The fish will want to jump over the fall of water between B1 and B2 which makes them stay in the area. Under these conditions, the system is started even with a single specimen (fish). The two mobile pallets will be folded for a maximum of five seconds afterwards automatically closing due to the winches and metal springs. There are visiting chambers for both the rectangular parallelepiped room below B2 and the space where the rectangular parallelepiped basin is located horizontally. All components of the system must be non-corrosive and of good quality. In winter the ichthyofauna sensor can be de-energized which will cause system shutdown. To clean or repair the system, close the valve. Also in winter this system may close. This system is fully automatic and can be applied to other dams of other structures and design. 
3. The lower Bistra Mărului River is significantly fragmented by a four m high spill threshold (Fig. 24).

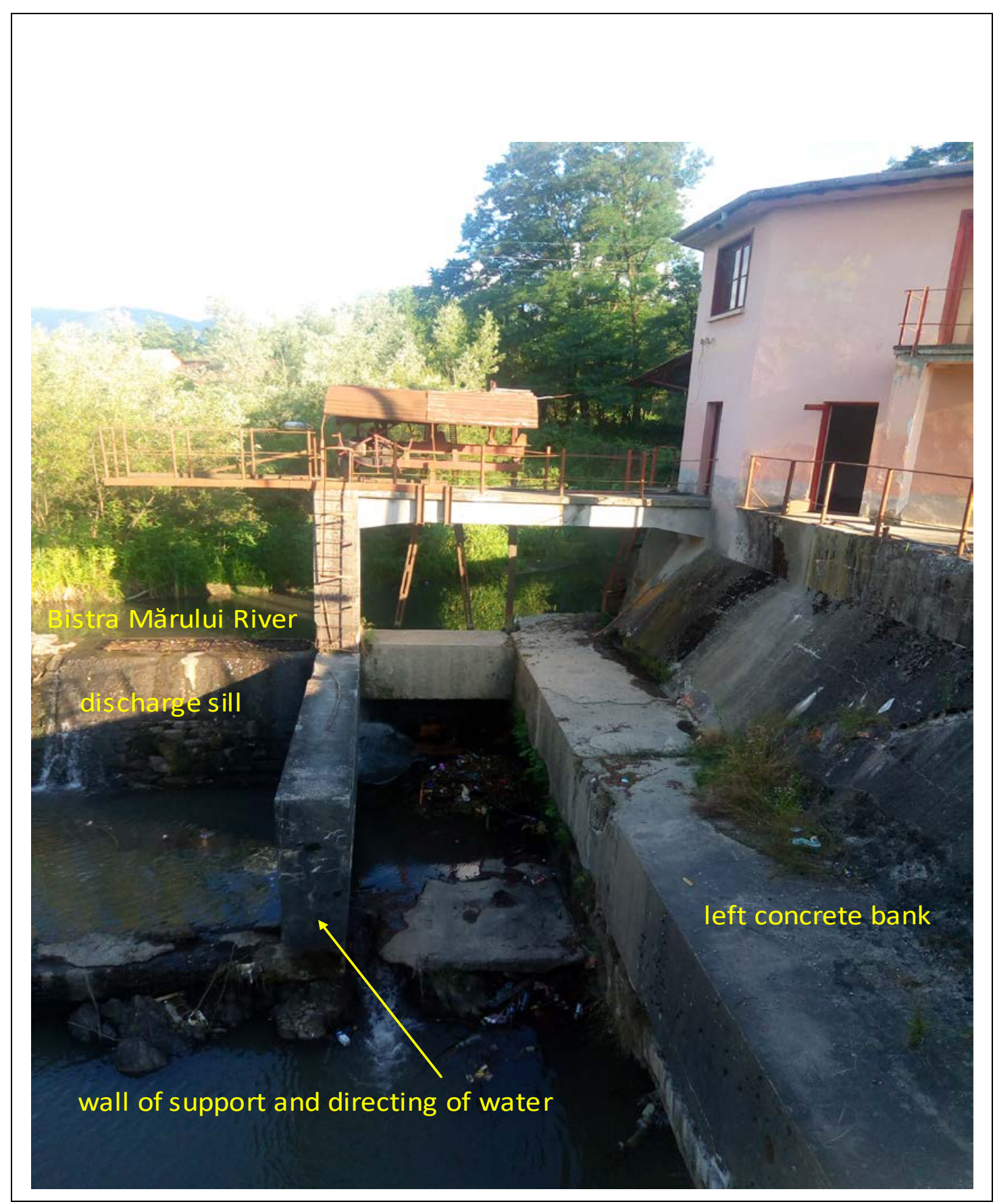

Figure 24: The discharge sill

of the lower Bistra Mărului River sector. 
Two rectangular breaches of $40 \mathrm{~cm}$ width and $60 \mathrm{~cm}$ height must be made, one made in the barrage near the supporting and watering wall, and the other in the water support and guide wall (Fig. 25). Between the two breaches on the support wall and the water direction, a rectangular channel (module 1) of concrete piles of three $\mathrm{cm}$ thick (Fig. 25) is fastened by means of metal dowels and horizontal bars. Thus the two breaches are directly related. The water from the first breech reaches the second bay through module 1 (Fig. 25). A second rectangular channel (module 2) is also attached to the downstream end of break 2 by three $\mathrm{cm}$ thick concrete sheet pile and having the same dimensions as the first module (Fig. 25).

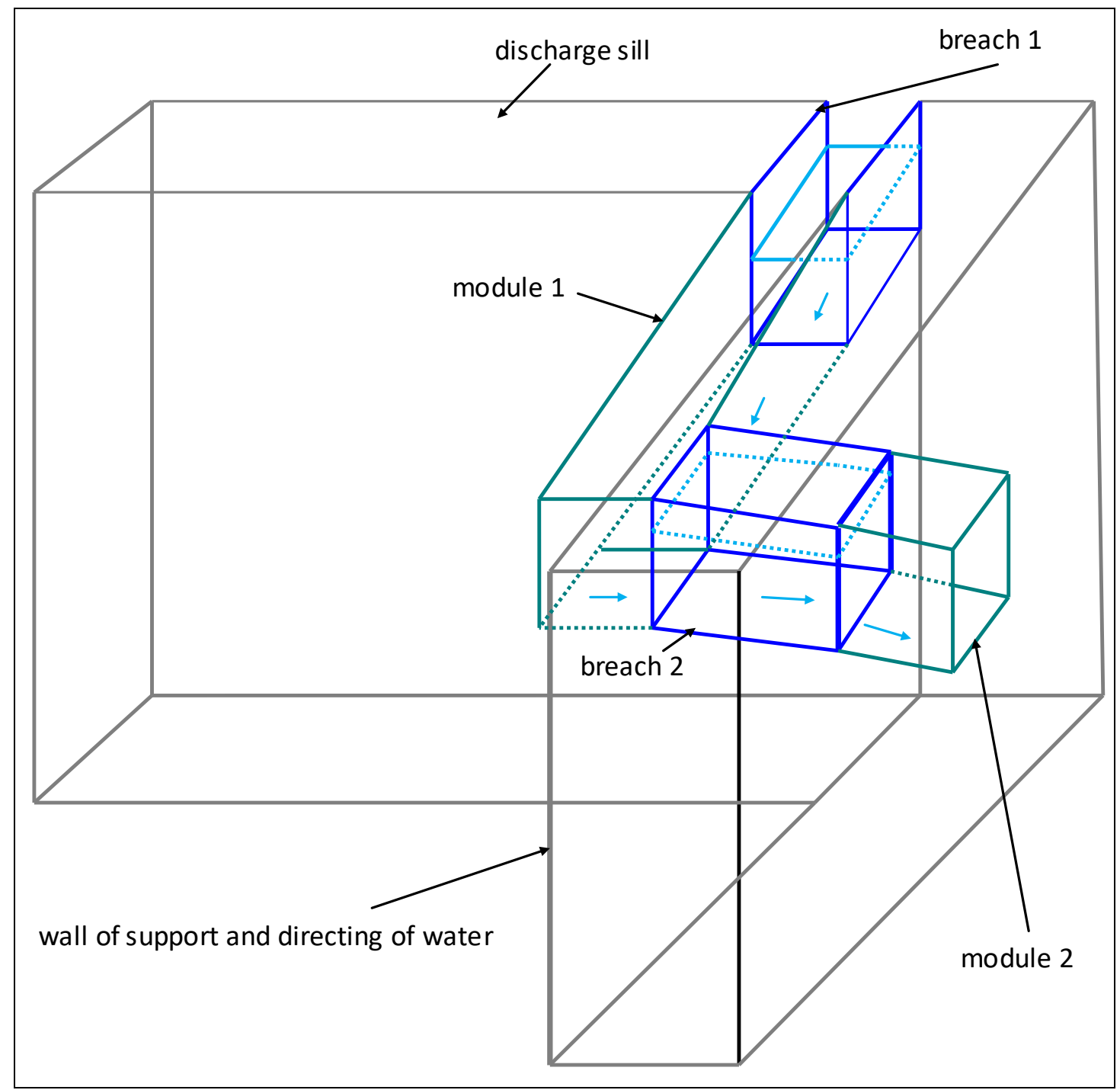

Figure 25: Positioning the two breaches and the two modules. 
Module 2 continues to the right bank. On the right bank concrete module 2 connects to module 3 (Fig. 26).

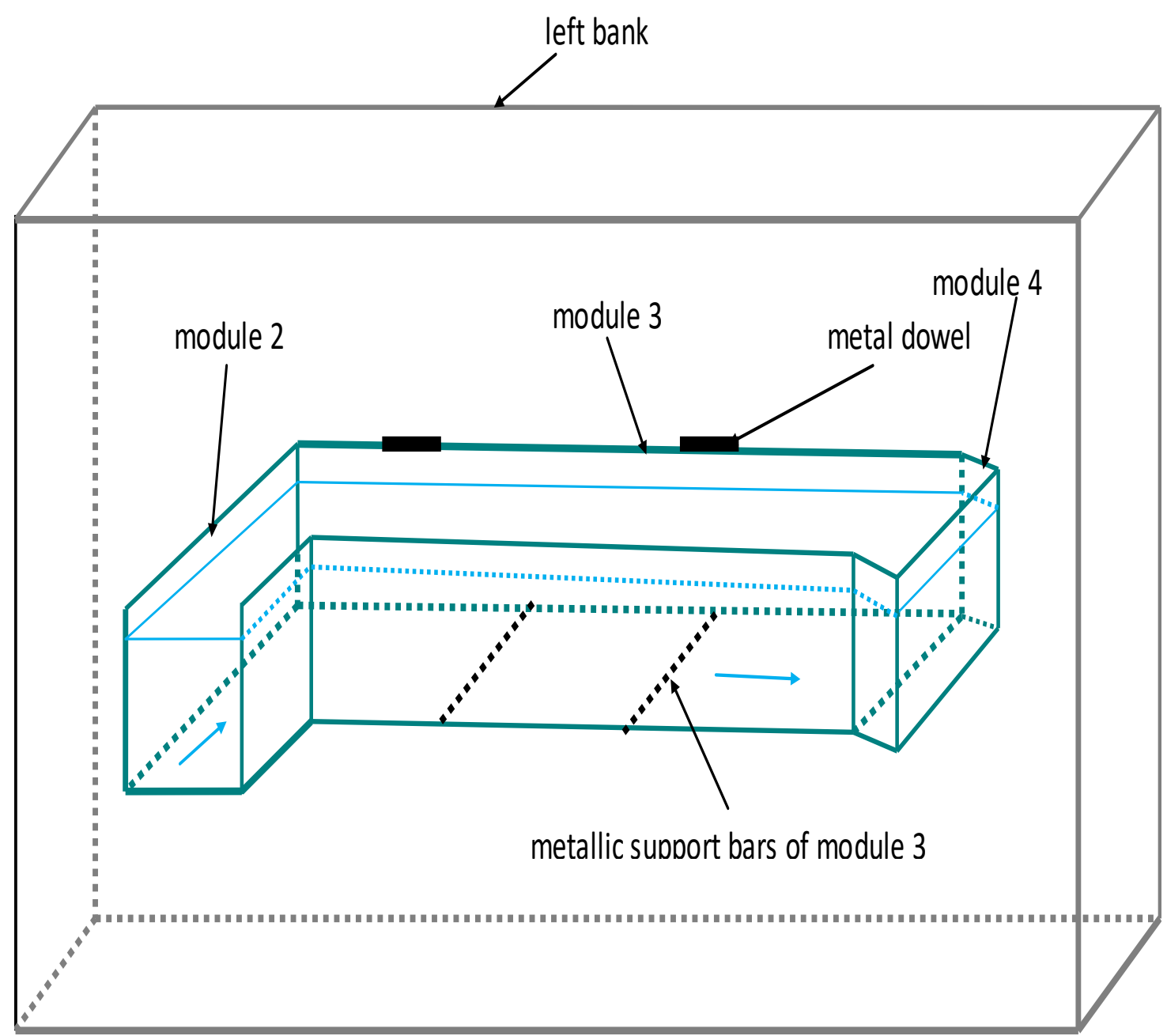

Figure 26: Positioning modules 2, 3 and 4.

From the downstream end of Module 3 will start Module 4 which will have the downstream end in two hydraulic junction basins of five $\mathrm{cm}$ (Fig. 27). The slope of all modules is smaller than the river slope in the study area. At the upstream end of the beam 1 , a metallic grid in the semicircle, which stops the pet or floating wood material, will be fixed to the spill threshold. The grill will be $40 \mathrm{~cm}$ thick and will rise above the water at least five $\mathrm{cm}$. The system has a durable structure and can withstand flooding. 


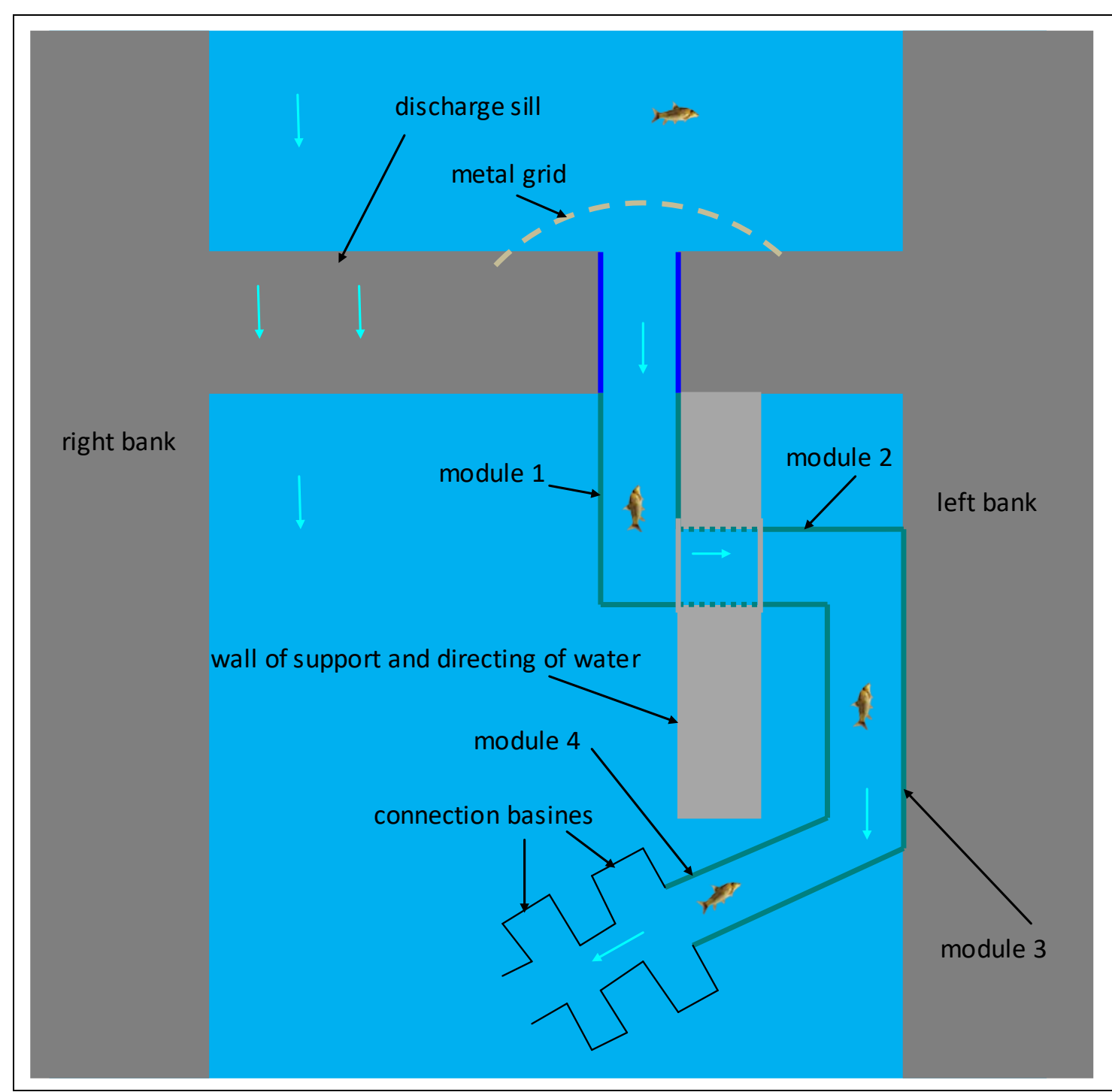

Figure 27: Positioning of the two connecting basins.

\section{CONCLUSIONS}

In a significant valuable from ichthyofauna conservation point of view basin of Timiş River, the Bistra Mărului River lotic habitats were heavily fragmented in the last 40 years by a large variety of hydrotechnical works categories (small $-0.20 \mathrm{~cm}$ to two $\mathrm{m}$ high, medium two to four $\mathrm{m}$ high and big - four to $125 \mathrm{~m}$ high), all of them builded in the communist period of Eastern Eurpe when river fragmentation and natural conservation values were concepts almost totally missing in the field of these works construction.

The Carpathian Fish Index of Biotic Integrity (CF-IBI) values variation in space and time reveal athropogenic induced negative effects on characteristic fish fauna integrity, from past (natural) to present (deteriorated) and from upstream (still natural from some points of view) to downstream (deteriorated). The Carpathian Fish Index of Biotic Integrity values decreased in the big majority of the river, often significantly and with a continuous decreasing trend. 
The most valuable and key fish species (Salmo trutta fario, Cottus gobio, Thymallus thymallus, Eudontomyzon danfordi, Eudontomyzon vladykovi, Gobio uranoscopus, Barbus meridionalis, and Condrostoma nasus) of Bistra Mărului River have declined significantly over the past two decades to the river fragmentation and water flow diminishing. Among them Thymallus thymallus and Gobio uranoscopus are now locally extinct (in Mara, Bistra Bouţarului and Bistra rivers is still present), and Eudontomyzon vladykovi is critically endangered.

Among the selected key fish species decreasing trend, the appearance, colonization and thrive of some not sensible fish species (e.g. Squalius cephalus and Gobio gobio) populations underline too the "sad story" of an extremely valuable Carpathian river ichthyofauna from conservative point of view.

All the three ichthyological zones for Bistra Mărului River suffer significant structural changes and an obvious major shifting, with an obvious advance from downstream to upstream of numerous uncharacteristic fish species.

The lack of proper scientific and practical knowledge in aquatic ecology in general and of ichthyology in special, the economic past and present very high and sometimes not moral and legal pressure on local administrations and environmental agencies, the so called political decisions and some inabilities in manage natural resources and services determine a continuous qualitative and quantitative degradation of habitats and related biocoenoses components, including obviously the fish.

All the technical solutions proposals of this research paper for the present hydrotechnical work negative effects diminishing are both functional and aesthetic in the hydrotechnical constructions in the study. These engineering solutions are designed to have a minimal negative impact during construction, designed to function without human intervention over a long period of time and without electricity consumption (for the three spill thresholds) and with minimal energy consumption for the system at the Poiana Mărului Dam. All the proposed solutions do not affect the structure of the hydro-technical constructions in the study area. The proposed solutions will make an important contribution to the recovery of local fish habitats and communities.

For the conservation of all key species (Salmo trutta fario, Cottus gobio, Thymallus thymallus, Eudontomyzon danfordi, Eudontomyzon vladykovi, Gobio uranoscopus, Barbus meridionalis, and Condrostoma nasus), the ecological status can be improved only if fish passage is improved. Furthermore, no other human constructed obstacles should be constructed (overfishing, mineral exploitation of the riverbed, pollution, banks and riverbed modifications, riverbanks ligneous vegetation deterioration, etc.).

The Bistra Mărului Basin ichthyofauna remains one of the most threatened in the Carpathians and urgent professional management care should be implemented! 


\section{ACKNOWLEDGEMENTS}

The first author of this research paper is grateful for the chance to spend a wonderful childhood and teenaging in Bistra River Basin, and hope that a proper future ecological management of this basin will offer a similar chance for the coming generations of kids and not only. 


\section{REFERENCES}

1. Aadland L. P., 2010 - Reconnecting Rivers: Natural Channel Design in Dam Removals and Fish Passage, Minnesota Department of Natural Resources, First Edition, 196.

2. Acreman M. C. and Dunbar M. J., 2004 - Methods for defining environmental river flow requirements - a review, Hydrology and Earth System Sciences, 8, 861-876.

3. Badea L., Gâştescu P. and Velcea V., 1983 - Geografia României, Geografie fizică, Edit. Academiei, București, 652. (in Romanian)

4. Baumgartner L. J., Zampatti B. P., Jones M., Sturt I., Mallen-Cooper M., 2014 - Fish passage in the Murray-Darling Basin: Not just an upstream battle, Ecological Management and Restoration, 15, 28-39.

5. Bănăduc D., 1999 - Data concerning the human impact on the ichthyofauna of the upper and middle sectors of the Olt River, Transylvanian Review of Systematical and Ecological Research, 1, Edit. Universităţii "Lucian Blaga” din Sibiu, ISSN 1841-7051, ISBN 973-9410-693, 157-164.

6. Bănăduc D., 2000 - Ichthyofaunistic criteria for Cibin River (Transylvania, Romania) human impact assesment, Travaux du Museum National d Histoire naturelle Grigore Antipa, Bucureşti, 365-372, XLII.

7. Bănăduc D., Mărginean M. and Curtean-Bănăduc A., 2013 - Geographical and human impact elements influence on the fish fauna of the Olteţ River (Romania), Transylvanian Review of Systematical and Ecological Research, 15.2, The Wetlands Diversity, Edit. Universităţii "Lucian Blaga” din Sibiu, ISSN 1841-7051, 9-44.

8. Bănăduc D., Rey S., Trichkova T., Lenhardt M. and Curtean-Bănăduc A., 2016 - The Lower Danube River - Danube Delta - North West Black Sea: A pivotal area of major interest for the past, present and future of its fish fauna - A short review, Science of the Total Environment, 545-546, 137-151, DOI: 10.1016/j.scitotenv.2015.12.058, ISSN 0048-9607, eISSN 1879-1026.

9. Bănărescu P. M., 1964 - Fauna Republicii Populare Române, Pisces-Osteichthyes, XIII, Edit. Academiei Române, 959. (in Romanian)

10. Birk S., Bonne W., Borja A., Brucet S., Courrat A., Poikane S., Solimini A. G., van de Bund W., Zampoukas N. and Hering D., 2012 - Three hundred ways to assess Europe's surface waters: An almost complete overview of biological methods to implement the Water Framework Directive, Ecological Indicators, 18, 31-41, DOI 10.1016/j.ecolind.2011.10.009.

11. Burghelea B., Bănăduc D. and Curtean-Bănăduc A., 2013 - The Tmiş River basin (Banat, Romania) natural and anthropogenic elements. A study case - management challenges, Transylvanian Review of Systematical and Ecolgical Research, 15, The Timiş River Basin, Edit. Universităţii Lucian Blaga din Sibiu, ISSN 1841-7051, 173-206.

12. Cambray J. A. and Bianco P. G., 1998 - Freshwater fish in crisis, a Blue Planet perspective, Conservation Biology, 65, 1, 345-356.

13. Katopodis C., 1990 - Advancing the art of engineering fishways for upstream migrants, Proceedings International Symposium on Fishways, Gifu, 90, Japan, 19-28.

14. Kemp P. and O’Hanley A., 2010 - Procedures for evaluating and prioritising the removal of fish passage barriers: a synthesis, Fisheries Management and Ecology, 17, 4, 297-322.

15. Mallen-Cooper M and Brand D., 2007 - Non Salmonids in a salmonid fishway: What do 50 years of data tell us about past and future passage, Fisheries Management and Ecology, 14, 5, 319-332.

16. Oberdorff T., Tedesco P. A., Hungueny B., Leprieur F., Beauchard O., Brosse S. and Dürr H. H., 2011 - Global and regional patterns in riverine fish species richness: A review, International Journal of Ecology, http://dx.doi.org/10.1155/2011/967631.

17. Olopade O. A., 2013 - Preliminary observations on the Family Mormyridae in Oyan Dam lake (Nigeria), Transylvanian Review of Systematical and Ecological Research, 15.1, The Wetlands Diversity, 33-48. 
18. Rathert D., White D., Sifneos J. C. and Hughes R. M. 1999 - Environmental correlates of species richness for native freshwater fish in Oregon, Journal of Biogeography, 26, 257-273.

19. Richter B. D., Postel S., Revenga C., Scudder T., Lehner B., Churchill A. and Chow M., 2010 Lost in Development's Shadow: the downstream human consequences of dams, Water Alternatives, 3, 2, 14-42.

20. Rumana H. S., Jeeva V. and Kumar S., 2015 - Impact of the Low Head Dam/Barrage on fisheries - a case study of Giri River of Yamuna Basin (India), Transylvanian Review of Systematical and Ecological Research, 17.2, The Wetlands Diversity, 119-138.

21. Sala O. E., Chapin F. S., Armesto J. J., Berlow E., Bloomfield J., Dirzo R., Huber-Sanwald E., Huenneke L. F., Jackson R. B., Kinzig A., Leemans R., Lodge D. M., Mooney H. A., Oesterheld M., LeRoy Poff N., Sykes M. T., Walker B. H., Walker M. and Wall D. H., 2000 - Global biodiversity scenarios for the year 2100, Science, 287, 1770-1774.

22. Soolutayo V., 2012 - Effects of human activities on the biodiversity of a tropical man made lake (Esinminrin Dam), Transylvanian Review of Systematical and Ecological Research, 14, The Wetlands Diversity, 175-186.

23. Stavrescu-Bedivan M.-M., Aioanei F. T. and Scăeţeanu G. V., 2017 - Length-weight relationships and condition factor of 11 fish species from the Timiş River, Western Romania, Agriculture and Forestry, 63, 4, 281-285, Podgorita, Serbia.

24. Stiassny M. L. J., 2002 - Conservation of freshwater fish biodiversity: The knowledge impediment, Verhandlungen der Gesellschaft für Ichthyologie, 3, 7-18.

25. Telcean I. C. and Cupşa D., 2015 - Captive populations of fishes in the Crişul Repede River (Tisa River Basin), Pisces Hungarici, 9, 75-80.

26. Tockner K., Robinson C. T. and Uehlinger U., 2008 - Rivers of Europe, ISBN-13, 978-0-12369449-2 first edition, 728, ISBN: 9780123694492.

27. Voicu R. and Merten E., 2014 - Creating a system for upstream - downstream fish migration over the first and the second discharge sills downstream of Mănăştur Dam on the Someşul Mic River (Cluj Napoca, Transylvania, Romania), Transylvanian Review of Systematical and Ecological Research, 16.2, The Wetlands Diversity, 161-180.

28. Vörösmarty C. J., McIntyre P. B., Gessner M. O., Dudgeon D., Prusevich A., Green P., Glidden S., Bunn S. E., Sullivan C. A., Reidy Liermann C. and Davies P. M., 2010 - Global threats to human water security and river biodiversity, Nature, 467, 555-561, doi:10.1038/nature09440.

29. Voicu R. and Baki P., 2017 - Improving upstream and downstream fish passage at Retiş Dam on Hârtibaciu River - Sibiu County (Transylvania), Annals of Valahia University of Târgovişte, Geographical Series, 17, 1, 47-57.

30. Voicu R. and Brețcan P., 2014 - Solution for fish migration on the Someşul Mic River upstream downstream of Mănăştur Dam in Cluj Napoca, Annals of Valahia University of Târgovişte, Geographical Series, 14, 1, 125-132.

31. Voicu R., Costescu C., Voicu L. and Lengher-Bica N., 2015 - Solution for ichthyofauna migration upstream-downstream of the two spillways located near Mănăştur Dam on the Someşul Mic River in the Cluj Napoca City, Lakes, reservoirs and ponds, 9, 1, 43-55.

32. Voicu R. and Dominguez L., 2016 - Facilitation fish migration above the discharge sill located on the Ialomiţa River near cave Ialomicioara, Annals of Valahia University of Târgovişte, Geographical Series, 16, 2, 44-58.

33. Voicu R., Voicu L., Curtean-Bănăduc A. and Bănăduc D., 2017 - Restoring the fish fauna connectivity of the Hârtibaciu River - Retiş Dam study case (Transylvania, Romania), Acta Oecologica Carpatica, X.II, 73-86.

34. Voicu R., Bănăduc D., Baumgrtner L. J., Voicu L. and Curtean-Bănăduc A., 2018 Transylvanian Review of Systematical and Ecological Research, 20.1, The Wetlands Diversity, Upper Caraş River (Danube Watershed) fish populations' fragmentation - technical fish populations' fragmentation - technical rehabilitation proposal, 69-86, ISSN-L 1841 - 7051, ISSN 2344-3219. 